\title{
ON THE FOUNDATIONS OF A NEW APPROACH TO STATISTICAL MECHANICS OF CLASSICAL SYSTEMS
}

\author{
S. N. BAGGHI \\ Department of Physics \\ Concordia University \\ Montreal, Quebec H3G 1 M8 Canada
}

(Received March 31, 1980)

ABSTRACT. It has been known for a long time that the fundamental approaches to equilibrium and nonequilibrium statistical mechanics available at present lead to physical and mathematical inconsistencies for dense systems. A new approach, whose foundation lies in the more powerful statistical method of counting complexions, had been formulated which not only overcomes all these difficulties but also yields satisfactory physical results for dense 'hard sphere' systems as well as for systems containing charged particles for which a mathematically consistent theory cannot even be formulated if we follow the available formalisms. The specific computational techniques rely on the following four recipes which also are justified theoretically.

(i) The phase space ( $\mu$-space) is separated into configuration space and momentum space.

(ii) The configuration space is partitioned into cells of size b, the exclusion volume of Boltzmann. 
(iii) The partition function (pf) due to the kinetic energy is obtained directly from Planck's"Zustandssumme" pertaining to the kinetic energies of the individual particles.

(iv) Instead of calculating Gibbs' configuration integral, one obtains the average potential of the system from a suitable nonlinear partial differential equation (pde) and finally the "excess" free energy of the system due to the potential field alone by utilizing Debye-Hueckel's concept of ion-atmosphere and their technique for calculating the free energy.

Even in the linear approximation of the ion-atmosphere potential this method gives reliable results for both equilibrium and transport properties of fused alkali halides.

In order to emphasize that this new approach has a secure theoretical foundation and has also considerable advantages over all other existing methods, this review offers a few brief critical remarks about the limitations and inadequacies of the concepts used in the conventional treatments of classical statistical mechanics. Further, in view of the fact that the literature on the subject of DebyeHueckel (DH) theory of strong electrolytes is replete with many assertions, already disproved in the past, a brief review of the controversial aspects of this theory is also presented. The next paper will show that this new approach as well as the modified DH theory yields physical results for actual dense systems much more satisfactorily than those which could be obtained by any other avallable method. KEY WORDS AND PHRASES. Statistical mechanics, Thermal physics, Electrolytes, Plasmas.

SUBJECT CLASSIFICATION CODE. $0064,1150,1220$.

1. INTRODUCTION.

In recent publications, (Bagchi $[1-3]$ ), it has been proved that the fundamental approaches to equilibrium statistical mechanics available at present, namely, 
the distribution function approach and Mer's cluster integral technique, lead to mathematical and physical inconsistencies for dense systems. Bogoliubov's apprach [4] reduces both these two methods as well as nonequilibrium statistical mechanics to a single concept, namely, that of $\mathrm{N}$-particle distribution function $\mathrm{f}_{\mathrm{N}}\left(\mathrm{q}_{1}, \mathrm{q}_{2}\right.$, $\ldots, \mathrm{q}_{\mathrm{N}}, \mathrm{p}_{1}, \mathrm{p}_{2}, \ldots, \mathrm{p}_{\mathrm{N}} ; \mathrm{t}$ ) which obeys Liouville's equation. The conventional kinetic approach rests on the generalized Boltzmann equation which uses one-particle distribution function of a point particle of the actual physical system in $\mu$-space. In order to avoid terminological confusions, in this paper the system will mean the actually given macrosystem and its constituents, (atoms, molecules, ions, etc.), will be called particles. Further, we shall deal only with conservative systems with a given Hamiltonian and mutually interacting potential which depends on the distance between two particles.

Since in most cases one cannot solve Liouville's equation exactly, one is forced to adopt some sort of expansion scheme. Obviously, such a perturbation technique has a physical meaning only when the series is mathematically convergent, or at least proves to be asymptotically convergent. Now, all theories, both for nonequilibrium and for equilibrium phenomena, relying on the usual computational techniques lead to mathematically divergent results for dense systems. It is important to note here that Cohen [5] had shown that for systems with short range forces one cannot use the expansion scheme à la Chapman \& Enskog, (see Chapmann \& Cowling [6]), in powers of density.

For equilibrium phenomena it had been already established that even for "hard spheres" the virial expression, obtained either from the distribution function theory or from Mayer's cluster expansion scheme, diverges for high densities. The virial coefficients calculated from these theories ther fore cannot be accepted as correct in principle even for dilute systems where the convergence difficulties do not arise in practice because of the magnitude of the density. Further,

it follows from Bogoliubov's remarks, (see his oft 
quoted text [4]), that for a system of charged particles a mathematically consistent scheme cannot even be formulated if one follows the traditional techniques. It is worth noting here that the original Debye-Hueckel (DH) theory, as proved before, (see Bagchi [7]), cannot be accepted even as a limiting theory. As long as one uses Boltzmann distribution in the field of electrostatic forces alone, one is implicitly using the model of point charges. Kramers [8] had proved from general considerations, and without Invoking the Ion-atmosphere concept, that a system of point charges leads to absurd results. Gronwall [9] proved mathematically that the situation is not changed if one introduces the usual boundary conditions on the surface of the Ion having a finite radius in the original Poisson-Boltzmann (PB) equation of $\mathrm{DH}$ theory of strong electrolytes [10]. Although Bogoliubov [4] obtained a formal expression for the limiting law of $\mathrm{DH}$ theory, his comment clearly points out that this does not imply that the original DH theory is a mathema tically consistent theory even in the limiting case of infinitely dilute solutions. He writes, for "Coulomb interaction, higher approximations would not be meaningful since the degree of divergence at the transition to small $r$ would increase. This divergence is due not only to the fact that a system of material particles in Coulomb interaction cannot be in a state of statistical equilibrium. If we include an additional potential for short-range forces this will make the situation only worse, pp. 41-42"' Consequently, one cannot formulate a consistent theory if one follows the traditional methods. But the situation is radically altered if one introduces the exclusion volume in the distribution function itself, (cf. ref. [3]). The theory then becomes entirely self-consistent.

Consequently, it can be safely asserted that all existing theories of equilibrium and nonequilibrium statistical mechanics will lead to divergence difficulties unless the mathematical techniques employed are suitably modifled. By the way, quantum mechanical treatments also suffer from these divergence difficulties.

I would like to emphasize here that these difficulties, In most cases, are 
not due to the inherent defects of the fundamental theory underlying the foundation of statistical mechanics. They arise principally from the fact that computational techniques do not take into account directly the physical realities, particularly the fact that all real physical systems contain particles which have "hard cores". The 'hard core' pertaining to a single particle cannot be encroached upon by any other particle of the system. They are therefore the exclusion volumes of Boltzmann. By incorporating this "hard core" in an analytic interaction potential of the Hamiltonian one cannot avoid these exclusion volumes. Simple considerations of the total energy and stability conditions would show that the centroids of two particles cannot approach a closer distance than a certain minimum distance $r_{0}$. Moreover, for a hard sphere system it is known that the total energy of the system is $\frac{3}{2} \mathrm{NkT}$; where $\mathrm{N}$ is the number of particles, $k$ is Boltzmann's constant and $\mathrm{T}$ is the absolute temperature. Obviously, expressing the Hamiltonian in the form, as is usually done,

$$
H=\sum_{i=1}^{N} p_{i}^{2} / 2 m+U(r) ; \quad U(r)=\left\{\begin{array}{l}
\infty \text { for } r<r_{0} \\
0 \text { for } r>r_{0}
\end{array}\right.
$$

has got no physical meaning. Nevertheless, if one expresses the Hamiltonian in this way merely as a mathematical artifice for convenience of calculation, then, to be consistent, in the processes of integration we have to take account of the fact that the volume available to a particle or to a cluster of s-particles is not the total volume $V$ but $[\mathrm{V}-(\mathrm{N}-\mathrm{s}) \mathrm{b}]$, where $\mathrm{b}$ is the exclusion volume of a particle.

There are three ways of incorporating these exclusion volumes which could overcome these difficulties. One is to include them directly in the Hamiltonian structure of "point" mechanics. We must, however, remember that we are dealing not with infintte total energies and singular potentials but with a large number of moving nonholonomic constraints in the form of inequalities or with moving infinite potential barriers as boundary conditions. 
The second method is to use the conventional theories of statistical mechanics but in the evaluation of the relevant integrals one should note that the entire volume is not available to a particle.

The third alternative is the easlest and the most effective way to get concrete physical results. It also provides a mathematically consistent statistical mechanical theory for all densities and for all systems. In this method one uses these exclusion volumes directly into the distribution function itself as in this new approach, (cf. [3]). The success of this method is mainly due to the fact that the phase space $(\mu$ - space) is partitioned into configuration space and momentum space.

It is generally agreed that the two fundamental approaches to equilibrium statistical mechanics cannot be applied to liquids. Frenkel [11] therefore tried to develop a kinetic theory of liquids. But major efforts were directed to the constructions of a model for the liquid with various assumptions for which Gibbs configuration integral can be calculated at least to a reasonable approximation. But all the so-called "model theories", except the significant structure theory of 1iquid proposed by Eyring et al [12], fail to predict satisfactorily the thermodynamic properties of the liquid, (cf. Muenster [13]). Model theories, like Lennard-Jones and Devonshire theory of liquid, "cell volume" and "hole" theories of Eyring et a1, the "tunnel" theory of Barker and various refinements and modifications of these model theories, no doubt deal with cells in the physical space. The fundamental basis of these theories, however, rests on Gibbs' phase integral. In fact, they are trying to calculate the configurational integral by further assumptions. The exact evaluation of the so-called "communal" entropy still remains a vexed and unsolved problem. The concept of cells in the configuration space is used mainly to split the "configuration partition function" into a product of identical similar integrals, one for each particle. The partial 
success of these model theories depends essentially on the fact that by using the cells in physical space, they had indirectly taken the exclusion volume, (albeit in an ad hoc way), into the formalism thus avoiding the divergence difficulties encountered in the integration processes of the conventional fundamental approaches.

It should however be noted that these "model theories" have nothing in common with the principles and techniques used in this new approach. The cells in this approach are cells of the phase space $\left(\mu\right.$ - space) having the size $h^{f}$ for a single particle with $\mathrm{f}$ degrees of freedom. h is Planck's constant. Only for convenience of calculation and for obtaining useful concrete physical results, this cell volume $h^{f}$ is expressed as a product of $b$, the cell volume of the physical space, and $\mathrm{h}^{\mathrm{f}} / \mathrm{b}$, the size of the cell in momentum space. Further, the volume of the cell b is to be identified with the exclusion volume of Boltzmann and not with the average volume per particle or the "free volume" of model theories. As proved previously, [cf. ref. [1]), if we follow Boltzmann's procedure to calculate statistically the average volume per particle, together with the virial coefficients given in the literature we come to the absurd physical result, namely, the average volume per particle becomes negative for dense systems. Of course, for dilute systems where $\mathrm{Nb}$ can be neglected in comparison to the total volume $\mathrm{V}$, the difficulties will not arise. Nevertheless, In principle the difficulty will remain. Perhaps, this is caused by the inaccurate virial coefficients rather than Boltzmann's method of expressing $b$ in powers of density.

The exclusion volume $b$ has a definite value for a particular system. Physically, its meaning is also clear and unequivocal. b represents the volume surrounding a single particle within which no other particle can ever enter. For pure simple liquids, it will correspond to the volume unencroached upon by other particles, the first neighbours. It is extremely difflcult to determine exactly the 
appropriate value of $b$, even for a simple system, which would lead to overall simultaneous agreement with all the thermodynamic properties of the system. We sha11 discuss this question in details in the next paper.

Further, the computational techniques of this new approach are fundamentally different from all other approaches available at present.

It might be pointed out here that the partitioning of the phase space was systematically used first by Dutta [14], (at the suggestion of (1ate) Prof. S. N. Bose), in dealing with the equilibrium statistical mechanics of hard sphere systems and of imperfect gases. Although his treatment showed that this separation of phase space is theoretically consistent, his method could not yield any concrete physical result, mainly because of the fact that one could not calculate the average potential of the system, the central problem of equilibrium statistical mechanics. The only feasible way to calculate this average potential is to utilize $\mathrm{DH}$ technique and to solve the corresponding nonlinear pde, (see Bagchi [7]).

Significant structure theory of liquids starts from the assumption that even a mono-phase macroscopic liquid consists of a mixture of perfect crystals and ideal gases, their relative proportions being determined by some empirical physical data such as the excess volume at the melting points. The pf of the 1iquid is then expressed as a product of the known crystalline pf and pf of ideal gases. It is obvious that the basic assumption of this theory is physically untenable. Nevertheless, it is interesting to note that this physically absurd model, though not in its literal sense, can be justified, (see Bagchi [15]), from the standpoint of the unified kinematic theory of diffraction by matter of any kind, (see, Hosemann \& Bagchi [16], Bagchi [17]). In this theory, from a single expression for the scattered intensity function $\vec{I}(\vec{b})$ one obtains not only the usual expressions for crystalline reflexions, liquid-1ike reflexions, amorphous scattering but also for the intensity scattered by substances which cannot be so rigidly classified. 
The diffraction pattern depends essentially on the distances between the scattering centres and their fluctuations, that is, on the socalled "distance statistics function $z(\vec{x}) "$. From the standpoint of diffraction, a liquid can be looked upon as an aggregate (clusters) of distorted microcrystallites. These distorted microcrystallites play the role of crystalline phase and the intercluster space that of ideal gas in the model underlying the significant structure theory.

Since our task here is to formulate a fundamental approach to statistical mechanics, model theories will not be discussed any further. Before we can attack the problem of dense systems satisfactorily and introduce further refinements in this new approach, we have to understand why existing theories fail not only to yield satisfactory physical results but become physically meaningless and mathematically inconsistent.

It is generally admitted that all existing theories fail conspicuously to predict rellable results for liquids and to explain first order phase transition which includes critical phenomena of liquid-vapour transition. These facts compel us to assume that the following two factors are principally responsible for the inadequacies of existing theories:

(i) The theoretical methods, though based on sound principles, lead to divergence difficulties because the essential physical reality, namely, that every physical system contains particles with "hard cores", has not been taken into account in computational techniques.

(ii) Some vital structural parameters of the liquid state have not been incorporated in the formalism of the theory.

More than 30 years ago, the author successfully tackled the problem (i) for a system of charged particles by modifying the original DH theory with the introduction of an empirical new distribution function instead of the Boltzmann distribution and by using the nonlinear ion-atmosphere potential, (see Bagchi [18]). The striking success of this method convinced the author that there must be a way to 
derive rigorously from statistical mechanical considerations a similar distribution function. At the suggestion of (late) Prof. S. N. Bose, the author in collaboration with Dutta derived rigorously an appropriate distribution function in which the exclusion volumes were directly incorporated in the distribution function itself, (see Dutta \& Bagchi [19], Bagchi [20]). Nevertheless, there are several difficulties with this distribution function as will be discussed later. Anyway, this modified DH theory proved to be mathematically consistent contrary to the original $\mathrm{DH}$ theory. Since then various workers had shown that the modified DH theory yields reliable results not only for equilibrium properties but also for the transport properties of solutions of strong electrolytes, (see references in [21]). The German school used only the linear approximation of the ion atmosphere potential and asingle value of the ionic radius ' $a$ ' and the single value of b. Consequently, in order to obtain good fit they had to use sometimes these values which are physically unrealistic. The Calcutta school, however used the actual ionic radii $a_{ \pm}$as well as different values of the exclusion volumes, $b_{+}, b_{-}, b_{+-}$of Dutta-Bagchi [19] distribution function as well as its approximate version, the formula used by the German school. This approximate formula was also derived afterwards by Wicke \& Eigen [22] from considerations of chemical equilibrium between sites occupied by hydrated ions and vacant sites. The results of the Calcutta school showed that one can get satisfactory results for activity coefficients by using the nonlinear potential and physically acceptable values of ionic radii and exclusion volumes. They calculated the nonlinear ionatmosphere potential and the corresponding free energy by the method used previously by Bagchi [18]. The nonlinear potential at the surface of the fon was obtained by Bagchi [18] by fitting the solutions of the modified PB equation for the two extreme cases of the potential, namely, $\lambda \rightarrow \infty$ and $\lambda \rightarrow 0$, at a certain point where the two solutions would smoothly fit into each another. In order to come to a definite conclusion about the merits of the ion-atmos- 
phere theory, the author [23] undertook an exhaustive comparative study of the problem with different distribution functions and different parameters and by using the exact analytic nonlinear solution of the generalized nonlinear pde of PoissonBoltzmann type $\nabla^{2} \lambda=f(\lambda)$, (see Bagchi \& P1ischke [24]). These voluminous results proved definitely that whereas the original $\mathrm{DH}$ theory cannot even lead to the known qualitative features of concentrated solutions of electrolytes, the modified DH theory can offer satisfactory results for activity coefficients throughout the concentration range by taking correct values of the exclusion volume. It might however be noted that the "fit method" does not give accurate results for the actual nonlinear potential. For example, for $a=1 \mathrm{~A}^{\circ}, \mathrm{b}=\frac{4}{3} \pi \mathrm{a}^{3}, \mathrm{c}=5 \mathrm{~N}$, the exact sol ution gave $\lambda=3.6$ whereas the fit method produced the result $\lambda=8.4$. These resu1ts are as yet unpublished but part of them will be communicated to this journal in the next paper. Recent work of the author on fused alkali halides (see [3]) and preliminary investigations on their transport properties proved beyond doubt that this new approach offers the only feasible technique for a system of charged particles which is mathematically consistent and also yields reliable concrete physical results.

In order to search for the missing essential structural parameters of the $1 i-$ quid state, the author had the privilege of discussing his ideas with (1ate) Prof. Max von Laue who then kindly asked him to work in his laboratory to develop an adequate diffraction theory. There in collaboration with Hosemann, a much more ambitious programme was accomplished. During 1951-56 we had succeeded in developing the most comprehensive kinematic theory of diffraction available at the present moment. This was reported in our monograph [16]. All the problems of kinematic diffraction phenomena had been resolved, at least in principle. They also offered some vital information relevant to the methodologies of statistical mehcanics. For example, this unified theory for the first time clarified the exact meaning of the radial distribution function $(R D F), 4 \pi r^{2} g(r)$, the assumptions underlying Zernike-Prins theory of liquids, the various factors which play significant roles in the small angle scattering and Ornstein-Zernike Theorem. Nevertheless, the structural parameters necessary to formulate a satisfactory statis- 
tical mechanical theory of simple liquids, which could also throw some light on the nature of first order phase transition at the atomic level, were not revealed until recently. Detalled discussions on the role of the Unified Kinematic theory of diffraction to the problems of liquids and of phase transitions had been discussed in my lectures [25]. Unfortunately, they are as yet unpublished. A short exposition of the unified theory together with the correct method of analysing RDF has been given in Bagchi [17]. Part of the new results about the structural parameters of liquids have been published in several papers, (see refs. [15], [2628]). Since results obtained from this unified theory are useful for formulating a correct statistical mechanical theory for the unsolved problems, section 2 summarizes some of the conclusions which are relevant for problems of equilibrium statistical mechanics.

Finally, in order to appraise correctly my critical remarks and comments about the drawbacks of the existing theories, it is necessary to state here very brief1y my opinions about an acceptable physical theory which, I belleve, are shared by a majority of mathematical physicists.

According to my opinion, an acceptable phyiscal theory should be judged by the following criteria:

(i) It should contain least number of independent postulates and incorporate important primary physical concepts in the structure of the theory. (ii) It should not lead to internal inconsistencies which necessarily mean that the mathematical model on which a physical theory is constructed should not lead to divergences, even if the theory can predict very accurate verifiable results. In the latter case, if the foundation of the theory is erected on a secure basis, one has to find exact mathematical methods to eliminate these divergences. (iii) Since a model, physical or mathematical, always contains some assumptions and ignores some pertinent factors, it is inevitable that the calculated values will not agree exactly with those obtained experimentally. But they must be"rel- 
lable". But the converse is certainly not always correct. A typical example of this is offered by the fact that the original DH theory cannot be accepted even as a limiting theory, though the calculated and experimental values agree satisfactorily for very dilute solutions.

(iv) No physically observable function can be infinte. The mathematical functions exhibiting discontinuities are smoothed out by the precision function of the measuring apparatus. The observable function $A$ is really a functional obtained by the convolution product of the mathematical function $f$ with the "precision" function $g$ of the measuring apparatus. That means, $A=f * g$, (* symbol for convolution product). Consequently, a large number of mathematical functions, (the socalled "function complexes"), may represent a single physically observable function. The so-called "Function Algebra" of Hosemann \& Bagchi [29] which connects the mathematical functions with the given physically observable function had been proved to be mathematically equivalent to Schwartz-Mikusinski - Temple approach of generalized functions, (see Ghosh [30]). But for many physical situations, part1cularly in the phenomena of small angle scattering and in phase transition and in the actual calculation of Fourier transform of a given function, the function algebra has many practical advantages over the concept of generalized functions. (For a brief exposition of function algebra, see ref [16] Ch. V and the Appendix). It is worth noting here also the pertinent remark of A. Sommerfeld: The art of theoretical physics lies in making appropriate approximations.

Consequently, one should not discard "hard sphere" model as unphysical. In fact, it can safely be assumed that all the fundamental properties of statistical mechanics which can be derived from this model will apply equally well, at least qualitatively, for real substances with short range attrative forces, in particular for inert elements like argon. Anyway, this hard sphere model is much more physically realistic than ideal gases for which, as is well known, all transport properties become infinite. 


\section{SUMMARY OF THE CONCLUSIONS OBTAINED FROM UNIFIED KINEMATIC THEORY OF DIFFR-} ACTION.

(i) On The Nature of The Liquid State.

Detailed and correct analysis of $\mathrm{RDF}$ obtained from diffraction data and the comparison between the experimental $g(r)$ and theoretical $\overline{n_{2}(r)}$ shows that even a simple liquid like argon at the triple point contains densely packed microclusters of approximate linear dimension $90 \mathrm{~A}^{\circ}$, (see [15]). This conclusion is also in conformity with the old idea that liquids consist of cybotactic groups and droplets (cf. Ter Harr $[31]$ ).

Liquids, particularly near their melting points, must therefore have to be considered as a collection of small thermodynamic systems. At the atomic level, the liquid is inhomogeneous from the point of view of mathematical statistics. One cannot therefore describe the macroscopic system with the help of a single statistics. In this connection one should particularly note the concept of "generalized lattice" and clusters of such lattices, the fundamental concept on which the unified theory was built up, (cf. refs [16], [17]). As the temperature rises, the statistical inhomogeneity vanishes gradually mainly due to random orientations of the micro-clusters, (see refs. [15], [26], [27]).

If one desires to follow the ensemble concept, then the liquid state should have to be studied with the help of the generalized ensemble whose pf $\gamma$ is given by

$$
\gamma=\sum_{i, \vec{N}, \vec{x}} \exp \left[\left(-\vec{X} \cdot \vec{x}+\vec{N} \cdot \vec{\mu}-E_{i}\right) / k T\right]
$$

where the independent variables are $\vec{X}, \vec{\mu}, T$, (cf. Hill [32] p 72). It is to be noted also that for a collection of small systems the second moment alone would not suffice to calculate the average properties of the system itself.

(ii) The Properties of Experimenta11y obtained RDF.

The function $g(r)$ obtained from scattered intensity is proportional to the 
convolution square of the density function, i.e. $\rho(\vec{x}) * \rho(-\vec{x})$. In fact, it is the spherically symmetric distance statistics function $z(\vec{x})$ of the unified theory. $g(r)=g_{0}(r)+\frac{1}{\bar{v}}=\bar{z}(\vec{x})-\delta(\vec{x}-0)=\bar{\rho} \bar{n}_{2}(r) ; \quad(\delta \equiv$ Dirac's delta function) Here $\bar{v}$ is the average volume occupied by a particle, $\overline{n_{2}}(r)$ is the average pair correlation function. The symbol represents the function averaged over all orientations in space. For Kinematic Theory, the scattered intensity corrected for all factors which do not depend on the density function gives the intensity function

$$
I(\vec{b})=R \cdot R *=\mathfrak{F}_{\rho}(+\vec{x}) \cdot \hat{\boldsymbol{F}} \rho(-\vec{x})
$$

$\mathrm{R}$ is the scattered amplitude, $\mathrm{R} *$ its complex conjugate

$$
Q(\vec{x}) \equiv \bar{y}^{-1} I(\vec{b})=\rho(\vec{x}) * \rho(-\vec{x})=\int \rho(\vec{y}) \rho(\vec{x}+\vec{y}) d v_{y}
$$

For a "point" structure, $\bar{Q}(\vec{x})=\frac{1}{\bar{v}} z(\vec{x}) \cdot s(\vec{x}) * s(-\vec{x})$

where

$$
z(\vec{x}) \quad \lim _{M \rightarrow \infty} \frac{1}{M} \sum_{m=1}^{M} \sum_{k=1}^{M} \delta\left(\vec{x}-\vec{x}_{m}+\vec{x}_{k}\right)
$$

$s(\vec{x})$ is Ewald's shape or stencil function. $\vec{j}, \vec{j}^{-1}$ represent Fourier transform and its inverse.* represents the symbol for convolution product. For the expression for a generalized lattice containing different kinds of particles in the cell, (sœ refs $[16],[17][15]), Q(\vec{x})$ is always proportional to $z(\vec{x})$ and therefore to $g(r)$. The latter is the space-time average of the pair correlation function. One must therefore note that the usual analysis of the experimentally obtained $g(r)$ based on Zernike-Prins technique of putting Gaussian function at the humps of the $g(r)$ function is both physically and mathematically wrong. The correct method of ana1ysis given by Bagchi [17] yields reliable physical results not only for known models but also for a large number of simple liquids, (see Bagchi $[15],[26],[27])$. One must deconvolute $g(r)$ correct $1 y$ to get $\rho(\vec{x})$. Consequently, $g(r)$ is very insensitive to the actual density distri- 
bution on the atomic scale. This is particularly true if the system contains densely packed clusters. But even in such cases, isothermal compressibility and expansivity are very sensitive to the available intercluster space. This is the main reason for the failure of distribution theory to predict reliable physical results even when the calculated $\bar{n}_{2}(r)$ agrees well with the experimentally obtained $g(r)$. In most cases, even for a simple liquid like argon, $\overline{n_{2}(r)}$ calculated from the distribution function theory differs even qualitatively from the experimentally obtained $g(r)$. But in the case of liquid argon near the triple point $\bar{n}_{2}(r)$ agreed fairly well with $g(r)$. Nevertheless, the calculated isothermal compressibility, which depends only on $g(r)$, differs by orders of magnitude from the experimental value. The problem had been discussed thoroughly by Bagchi [15].

One cannot and should not use $g(r)$ obtained from diffration theories, even when $g(r)$ is corectly deconvoluted, if one desires to predict reliable results for dense systems from the distribution function approach. $g(r)$ always gives only the space-time average of the density function and therefore of $n_{2}(r)$. Further, the unified theory proved, (see ref [16], Ch. V1), that one cannot even in principle hope to get either the exact pair correlation function $n_{2}(r)$ or the exact density function $\rho(\vec{x})$ from diffraction experiments. One obtains only the space-time average function within the so called "domain function", the Fourier transform of the "precision function" of the measuring apparatus. On1y for "primitive" liquids, i.e., liquids containing no clusters at all and satisfying some other conditions, (see ref. [16]Ch. XV1, p 558), the experimentally obtained $g(r)$, when inserted into the appropriate equation of the distribution function theory, may lead to reliable physical results. But it is extremely doubtful whether real liquids and even highly dense gases can ever be treated as systems containing no physical clusters. 
Since the technique of this new approach permits one to calculate the average potential of the system, it would enable one to calculate the pair distribution function necessary to evaluate thermodynamic properties from distribution function theory without using BBGKY hierarchy of equations and the superposition approximation.

(iii) Some Remarks About First Order Phase Transitions.

First order phase transition including critical phenomena is related to the structure of the liquid and of the dense gas. Since these two phenomena are occupying much attention of theorists now-a-days, it is hoped that the information extracted from a comprehensive diffraction theory might be useful to tackle these two as yet unsolved problems.

I would like to submit here again, (see Bagchi [28]), that the efforts to explain first order phase transition by using the criterion of the singularity of the pf of equilibrium statistical mechanics at the thermodynamic 1 imit have no physical and mathematical justification. Theoretical investigations of Van Hove [33] and of Yang \& Lee [34] proved convincingly that canonical and grand canonical ensembles cannot lead to such a singularity for a finite number of particles. And it has not yet been proved that such a singularity (in the real domain) exists at the thermodynamic 1 imit and whether analytic continuation is possible. The analogy of surface distribution of point charges is also not convincing, since phase transition manifests itself also as a bulk property and the particles have always finite sizes.

Further, experience tells us that sharp transitions take place for a system containing large but finite number of particles and this transition for very small systems is not so sharp, (see Hill [35]). Also, it is known that for any finite system, the pressure remains an analytic function throughout the entire region. This follows simply from the fact that the pf is always an analytic 
function for any finite N. Moreover, if we note the relation between mathematical functions and the physically observable function, it is evident that in the phenomenon of phase transition the experimentally observed isothermal parallel to the volume-axis arises from the fact that the true analytic behaviour of pressure is masked by the effects of measurement.

Again, even if the observed pressure (or any other variable) of the macrosystem remains constant, it does not necessarily imply that these variables remain the same for each of the subsystem (clusters), (cf.ref [35]). The pertinent values of the given macrosystem represent the space-time average values of these subsystems, where care must be taken to consider also the higher moments of the distribution function to calculate the average properties of the given macrosystem.

It is generally agreed that a study of the equilibrium theory cannot provide any information about the particular process by which the equilibrium is brought about. Consequent1y, it is obvious that only the laws of mechanics can enlighten us about the mechanism of phase transition. From the standpoint of foundation, the advantage of using the concept of thermodynamic limit to avoid boundary effects is offset by a much more difficult and subtle problem of an infinite dimensional Hamiltonian system. All the required properties of such systems have not yet been worked out, (cf. Chernoff \& Marsden [36], Sinai [37]). Also, it would be worthwhile to consider Fowler's remark [38] carefully: "An unenclosed system to which all space is accessible does not in general possess an equilibrium state about which anything significant can be said, p 9."

Further, physically also this is not necessary. As long as we are not interested in surface effects, we can safely ignore the boundary effects in equilibrium situations, since the so called "normal" properties of the macrosystem can be defined satisfactorily in a mathematically and physically consistent way without bringing in the concept of thermodynamic Iimit. In Jeans'[39] 1anguage, a 
property is a normal property of the system if that property is possessed by the system with negligible error in the whole of the accessible phase space except for a negligible fraction. That is, $\left[\mathrm{A}^{2}-\bar{A}^{2}\right]^{\frac{1}{2}} \ll|A|$ for any observable $A$. Fowler, (see [38], p. 11), uses the word "normal" as a synonym for short time average.

In statistical mechanics one often uses thermodynamic limit for mathematical convenience and to prove the equivalence of different types of ensembles. As will be pointed out later, it is not essential to use the ensemble concept of $\Gamma$ space for treating equilibrium phenomena. For transport problems the use of $\Gamma-$ space has got no physical significance. Consequently, this mathematical advantage as far as actual physical problems are concerned is illusory.

Diffraction studies had led to the conclusion that from the point of view of mathematical statistics first order phase transition is primarily governed by the conditions which lead to discontinuities associated with the transformation of a statistically homogeneous system, (eg. crystals and vapours), into an inhomogeneous entity, (on the atomic scale), the liquid.

Moreover, in phase transitions, (outside the critical region), we are dealing with the coexistence of two phases of different densities and different energy densities. Consequently, it is very unlikely that a single analytic expression for pf would decompose into two disjoint sets of particles. For a finite number of particles we know that this does not happen. And phase transitions do take place for a finite number of particles. I believe, only topological dynamics could explain such phenomena connected with the stability conditions and bifurcation of a dynamical system containing particles with hard cores.

It was suggested by the author [28] that for crystal-1iquid transition, a quantitative study of anharmonic lattice vibrations of large amplitudes of $\underline{f}$ inite sized particles, (which would obviously involve collision phenomena), under 
the influence of an external source (heat energy) is likely to demonstrate the existence of a jump discontinuity simflar to that of the well known behaviour of the Duffin equation. The old idea of Lindemann [40] about the mechanism of melting suggests that it is worth considering such lattice dynamics more carefully and thoroughly.

(iv) Critical Phenomenon of Liquid-Vapour Transition.

It is usually asserted that at the critical point many of the physical properties of the system become infinite. Most of these divergences arise from the fact that Ornstein-Zernike's generally valid relation about the observed small angle scattering is connected with the thermodynamic relation of isothermal compressibility via statistical mechanics of Gibssian ensemble. But it must be noted that compressibility physically means the relative change of volume with respect to pressure. Consequently, even if $(\partial \mathrm{p} / \partial \mathrm{v})_{\mathrm{T}_{\mathrm{C}}}=0 ;\left(\partial_{3}{ }^{2} \mathrm{p} / \partial \mathrm{v}^{2}\right)_{\mathrm{T}_{\mathrm{C}}}=0$, . they do not imply that the compressibility is infinite, since $(\partial \mathrm{p} / \partial \mathrm{v})_{\mathrm{T}_{\mathrm{c}}}<0$. Further, it is obvious that ensemble averages in $\Gamma$-space cannot throw any light on the internal fluctuation of densities and of atomic distribution and of correlation lengths prevailing within a particular member of the ensemble, the system itself, which is represented by a point. Dynamics of $\mu$-space, however, could reveal such microscopic details.

Detailed analysis of RDF of several samples of argon near the critical region published by Mikolaj \& Pings. [41] has definitely proved that near the critical region the average fluctuation of density distribution and average correlation length remain finite, (see Bagchi [27]). The latter conclusion does not even need any analysis. A glance at the published RDF curves would confirm this. This is also demonstrated convincingly by the mathematical model of the distribution of the nth neighbour for a known fluctuation of the nearest neighbour, (see Figs. $14(1-x)$ in ref [17]). This theoretical calculation also shows that 
crystalline, 1iquid-1ike and amorphous structures depend essentially on the distance statistics $z(\vec{x})$ of the generalized lattice, the starting point of the unified theory of diffraction. The monograph [16] proved this quantitively from studies of optical diffraction patterns of numerous carefully prepared models whose structure parameters were already known. All these studies proved that the average correlation length of the pair distribution function decreased as we approach the critical region and in the gaseous state giving continuous amorphous scattering it shrank practically to the diameter of the molecule or the closest distance between randomly packed "particles".

Ornstein-Zernike's theorem states that the small angle scattering is proportional to the fluctuations of the density distribution of the material. It. refers to the observable portion of the scattered intensity and does not include Debye's volume scattering which is always inaccessible. It remains valid irrespective of the nature of the fluctuation, namely, whether it is due to the dynamic motions of atoms, or is caused by the distribution of atoms in a "frozen" structure, (see pp. 220,553 of $[16]$, also p. 131, ref. [17]).

Detailed analysis of this theorem and the small angle scattering from the standpoint of the unified theory, (see Chs. VII, XI, XVI, XVII of ref. [16]), had led to the following conclusions:

(a) The small angle scattering as well as the background scattering depends on the fluctuations of the density distribution within a "particle" and on that of volume elements occupied by each particle, i.e., the relative distances between the centroids of the particles. That means, both polydispersity and density of packing contribute to these two phenomena.

(b) The sharp rise in the observed small angle scattering is mainly due to (ooo)relexions of the particles and not to the unobservable Debye's volume scattering. (c) It is known that at the critical point the scattered intensity becomes very pronounced. Nevertheless, the total amount of scattered radiation over the entire 
Fourier space remains constant, since

$$
\int I(\vec{b}) d v_{b}=N(v) \delta\left|f_{m}\right|^{2} d v_{b}=\text { constant }
$$

It includes also the unobserved central peak. As is well known Debye's volume scattering is proportional to $\overline{\mathrm{N}}^{2}$ and $\overline{\mathrm{I}}_{\text {obs }}$ as well as the total scattered intensity is proportional to $\overline{\mathrm{N}}$. ( $\left|\mathrm{f}_{\mathrm{m}}\right|^{2}$ denotes the structure factor).

The observed prominence in scattering is due to the fact that under normal circumstances the scattered radiation is distributed throughout the Fourier space, but in the neighbourhood of the critical region it is practically concentrated in the forward direction. That means, we are observing here essentially the (ooo)reflections of small clusters.

As already pointed out, diffraction experiments give only the space-time average values. Consequently, these results do not preclude the theoretical concepts that internal fluctuations of density is very large and correlations between atoms extend over a large macroscopic domain. In fact, preliminary investigations by the author, (not yet published), of the experimental data of Thomas \& Schmidt [42] on the scattered intensity of argon near the critical point indicate that the molecules within the system are in a flux. Number of atoms within a "particle" is constantly changing, clusters of different sizes are disintegrating and reforming continuously. This result is also in conformity with the old idea that in the critical regions small drops, (cybotactic groups), of liquid are being formed in one moment and in the next moment they breakup into smaller pieces including single molecules which migrate to the vapour phase, so that during the period of observation, the average density of the two phases remains the same. In a certain sense, this is also in conformity with the prevalent theoretical concept of large (but not infinite) local fluctuations of density and large correlation length.

The above remarks are pertinent to first order phase transitions and critical phenomena observed in crystal-1iquid-vapour transitions. In spite of many similar- 
ities, I think, these transitions ought to be distinguished from such transitions associated with order-disorder phenomena and other so called cooperative phenomena. The disorder in the 11quid states (and also for pure crystals), for example, Is caused by the fluctuation of the positions of nearest neighours originating from the nonlinear vibration of atoms. The correlation length is determined by this fluctuation of nearest nelghbour distances. The disorder is propagated through convolution operations as in the case of propagation of errors. But in the orderdisorder phenomena we are dealing with the probability of occupation of definite sites in a lattice by different particles, or the probability of the "spin" of the site. In structural phase-transition we are dealing with the minimum potential energy of different configurations of atoms.

I would like to conclude this section by the remark that in formulating a consistent theory of statistical mechanics of liquids and of phase transitions, including critical phenomena, one ought to consider the results obtained from the analysis of the most comprehensive diffraction theory. At the present moment, diffraction theories are the only means available to us to obtain detailed information about the distribution of atoms inside a macroscopic object. The statistical mechanical theory presented here, however, does not discuss the problems of phase transitions and of critical phenomena. It deals with the equilibrium and nonequilibrium statistical mechanics of a simple system existing in a single phase for all densities.

3. SOME RELEVANT COMMENTS ON EXISTING METHODS OF STATISTICAL MECHANICS

(1) On The Foundations of Statistical Mechanics.

The object of statistical mechanics is to study the average properties (and their flucuations) of a macrosystem containing a large number, (of the order of $10^{20}$ ), of fundamental entities, (atoms, molecules, lons, etc.), Interacting with one another in a definite manner. It is assumed that they can, at least in prin- 
ciple, be obtained from the laws of mechanics, classical or quantal. The time evolution of the system is governed by the laws of mechanics. Fundamenta1ly, it is the study of the groups of motion of non-random objects. Hence, stochastic processes,important as they are in predicting the collective behaviour of groups of objects, do not strictly belong to the actual discipline of statistical mechanics.

In practice, in order to obtain concrete physical results some sort of purely statistical hypothesis must be introduced because of the inherent nature of the problem, namely, large number of particles and ignorance of the exact initial conditions. In this connection it is worth quoting the perceptive remark of Schroedinger [43]: "The Individual case is entirely devoid of interest, whether detailed information about it is available or not, whether the mathematical problem it sets can be coped with or not. We realize that even if it could be done, we should have to follow up thousands of individual cases and could make no better use of them than compound them into one statistical enunciation".

Nevertheless, a rigorous formulation of the foundation of statistical mechanics demands that such probability concepts must be obtained from laws of dynamics. Although for practical purposes we have to introduce the concept of a priori probability, we cannot accept this as a fundamental independent postulate of statistical mechanics. Let us note here the remark of Fowler [38]: "If this ( a priori probability) is taken at its face value, the behaviour of the particles in the system must be according to the laws of chance and cannot be controlled by dynamical laws in ordinary space and time. This is a possible hypothesis but in the end hardly a satisfactory one. It is equally repugnant to classical and to quantum mechanfcs.p.9".

A study of Hamiltonian mechanics is essential, since it alone can lay the secure foundation of statistical mechanics by providing us with the qualitative behaviour of the macrosystem. If we wish to prove the ergodic theorem, if we 
desire to overcome "reversible" and "recurrence" paradoxes, if we want to know how nonstationary systems approach the equilibrium state, if we intend to understand the mechanism of phase transition, which obviously is connected with the stability and bifurcation of the system, I believe, we must study topological dynamics by incorporating directly exclusion volumes in the formalism, since it is evident that collisions between particles only can explain these phenomena. The recent work on ergodic problem, (see Sinai [44], Arnold \& Avez [45], makes this conjecture highly probable.

I do not share the point of view of some authors that the question of ergodic theorem is a redundant and a sterile one for the justification of the conventional methods of statistical mechanics. It is obvious that all observed macroscopic values represent the space and short-time averages of the appropriate functions. They give the "normal" properties of the macrosystem. In statistical mechanics one does not use directly Hamiltonian mechanics. One starts from the N-particle distribution function $f_{N}(\vec{q}, \vec{p}, t)$ which obeys Liuoville's equation, (cf. Mazo [45]), $\frac{\partial f_{N}}{\partial t}+\left[f_{N}, H\right]=0(\delta t) \rightarrow$ o for $\delta t \rightarrow o$

([] is the symbol for Poisson-bracket). Our physical results, the mean (ormost probable) values, are calculated by averaging over the distribution function of the phase space. As had already been stated by Tolman [47], if we want to avoid the ergodic problem we have to "introduce the hypothesis of equal a priori probabilities for different regions in the phase space that corresponds to the extension of the same magnitude, p. 60". Since the ergodic problem can now be assumed to be solved, one should not make thisumecessary additional hypothesis.

Perhaps it would not be out of place here to mention again some known features of the Liouville equation. 
(a) Contrary to Hamiltonian equations from which it is derived, in the Liouville equation $\vec{q}$ and $\vec{p}$ are not regarded as functions of time. The time evolution is described by the change in time of the function $f_{N}(\vec{q}, \vec{p} ; t)$ at a given point $(\vec{q}, \vec{p})$ of the phase space.

(b) $f_{N}$ is really a probability density function. But there is nothing probabilistic about the motion of $\mathrm{f}_{\mathrm{N}}$.

(c) Sometimes $f_{\mathrm{N}}$ is interpreted as the density of a cloud of points, assumed to be continuous, in $6 \mathrm{~N}$-dimensional $\Gamma$-space. A particular system, the actual physical system, is represented by a point of this $\Gamma$-space. The ensemble whose density is given by $f_{N}$ consists of a very large number of identical and independent systems which are "dynamically equivalent" but differs in their initial conditions. According to its physical interpretation given by Gibbs, all the macrosocopic observables including the total energy can vary from one replica to the other. One calculates only the mean (or most probable) value and its fluctuation. "Dynamically equivalent" means that all replicas have the same functional form of their Hamiltontans If we treat the ensemble realistically as a collection of finite sized systems suffering collisions, then $H$ in eq (3.1) can only mean the average energy of the cloud of $\mathrm{N}$ systems. Then eq. (3.1) would be valid if there exists an average potential for the average force.

For transport problems the ensemble concept and the interpretation of $f_{N}$ as a density distribution of point sets in $\Gamma$-space lose their usefulness. Gibbs also did not consider transport problems. His attempt to prove that non-stationary ensemble will attain stationary distribution in course of time did not succeed, because for this one has to consider collision phenomena. Obviously, an ensemble of non-interacting point set cannot produce the equlibrium state because of Liouville's theorem. 
Actually, one treats kinetic problems with the help of Boltzmann equation which represents much more physically meaningful concept of one-particle distribution of $\mu$-space. Perhaps Fowler [38] had this in mind when he rejected the ensemble concept. According to him, if we "demand certain sanity or physical reality in the initial postulates, Gibbsian ensemble appears to be weak, p. 7". Other drawbacks of the ensemble concept will be pointed out below.

It should be noted that statistical mechanics of $\mu-s p a c e$, in which we consider only the physical system of interest with the given total number of particles in a prescribed volume and having a given total energy, does not suffer from these conceptual difficulties of the $\Gamma$-space. In the Liouville equation $f_{N}$ represents the distribution of the actual particles in the phase space ( $\mu-s p a c e)$ of the actual system of interest and $H$ its exact Hamiltonian.

The main reason why in equilibrium statistical mechanics one rejected this meaningful $\mu$-space in favour of $\Gamma$-space lies in the fact that if we stick to the original scheme of Boltzmann and Planck, we can handle only a few systems where, to borrow the phrase of Schroedinger [48], we can speak of "private" energies of each particle. Consequently, even Fowler had to treat imperfect gases practically as a canonical system in which other particles of the given system behave as a thermal bath, (see Fowler [38] ch. VIII).

This technical difficulty can be overcome only by separating the phase space ( $\mu$-space) into configuration space and momentum space and distributing the particles in the configuration space over a layer of volume in which the potential energy can be assumed to remain practically constant as adopted in the new approach to be discussed below. 
(ii) Equilibrium Statistical Mechanics.

For equilibrium phenomena where only a few properties of the macrosystem are observable, one can use the ensemble concept and the distribution function in $\Gamma$-space. In fact, only one constant of motion, namely, the energy of the system, is necessary to develop fully the equilibrium statistical mechanics. As had been apt1y remarked by Schroedinger [48]: "There is only one problem in statistical thermodynamics, namely, the distribution of a given energy $E$ amongst $N$ particles of the system, p 5". In the language of quantum mechanics this means, the distribution of a collection of $\mathrm{N}$ particles of the system amongst the accessible stationary energy states $E_{i}$ of the system in such a way that the relation (3.2) is satisfied.

$$
E_{i}=E_{i 1}+E_{i 2}+\ldots+E_{i N}
$$

$E_{\text {is }}$ 's are energies of individual particles. All thermodynamic properties of the system can be determined from Planck's "Zustandssumme" (sum over states; pf.),(cf. Wilson [49] p.104)

$$
Z_{\text {system }}=\sum_{i} W\left(E_{i}\right) \exp -\left(E_{i} / k T\right)
$$

where $W\left(E_{i}\right)$ is the number of complexions of the system, i.e., the number of ways in which $\mathrm{N}$ particles of the system can be distributed so that relation (3.2) is satisfied.

In classical mechanics we can take $E_{i}$ as the given energy of the system and $E_{i s}$ to be continuously distributed. Consequent1y, for a system of identical particles without any internal structure, eq. (3.3) reduces to, (cf. [31], [32], [49]),

$$
\mathrm{Z}_{\text {system }}=\frac{1}{\mathrm{~N} ! \mathrm{h}^{3 \mathrm{~N}}}-\int \exp -(\mathrm{H} / \mathrm{kT}) \mathrm{d} \overrightarrow{\mathrm{q}} \mathrm{d} \overrightarrow{\mathrm{p}}
$$

Although this integral (3.4) represents pf in $\mu$-space, it is formally the same as the corrected Gibbs' canonical phase integral.

Gibbs introduced the ensemble concept as a postulate in order to connect 
the parameters of the stationary density distribution in $\Gamma-$ space to thermodynamic variables. In Boltzmann - Planck approach this is not necessary since the entropy $S$ is connected with the number of complexions $W$, the so-called Planck's thermodynamic probability, by Boltzmann's hypothesis

$$
\mathrm{S}=\mathrm{k} \ln \mathrm{W}
$$

The absolute temperature enters into the formalism by the maximization of the entropy subject to the conservation of the number of particles and the total energy and using the well known thermodynamic relation

$$
\left(\frac{\partial \mathrm{S}}{\partial \mathrm{E}}\right)_{\mathrm{V}}=\frac{1}{\mathrm{~T}}
$$

(The relation (3.5) can also be applied to non-equilibrium phenomena).

It should also be noted that the correction factor $\left(N ! h^{3 N}\right)^{-1}$ in eq. (3.4) can be rigorously derived only by quantum mechanical considerations. The volume of the cell in phase space cannot be determined from classical statistical mechanics. The real reason for the size $h^{f}$ of the cell in $\mu$-space for a particle with f degrees of freedom has been clearly explained by Ter Haar ( $[31], \mathrm{p} .60)$.

Since many text books still ascribe this cell size to the uncertainity principle (without noting the fact that the exact relation for the uncertainity principle is $\Delta \mathrm{p} \Delta \mathrm{q} \geq \mathrm{h} / 4 \pi)$, it would be desirable to mention here very briefly Ter Haar's explanation.

In quantum mechanical formalism, the probability density in q-space is given by $|\psi|^{2}$ which is normalized to unity. $\psi$ is Schroedinger's wave function. The wave function $\Phi(\mathrm{p})$ in momentum space is obtained by the Fourier tranform of $\psi$. Consequently, in quantum mechanics $\vec{q}$ and $\vec{p}$ are not independent. The natural variables in the momentum space is, however, the vector which is reciprocal to $\vec{q}$. Using de Broglie's relation we find that this variable is given by $\vec{p} / \mathrm{h}$. Since the wave function $\Phi(\vec{p})$ in the momentum representation is also normalized to unity, we get for a single particle with 3 degrees of freedom in the stationary state 


$$
\int|\psi|^{2} \mathrm{~d} \overrightarrow{\mathrm{q}}=1 ; \int|\phi|^{2} \mathrm{~d} \overrightarrow{\mathrm{p}}=\mathrm{h}^{3}
$$

Thus the probability that the particle will occupy a cell in the entire $\mu$-space is given by $h^{3}$.

The reason for the appearance of $N$ ! has often been ascribed to the nonlocalization of identical particles. But in classical mechanics we can distinguish the trajectories of each particle of the system, at least conceptually, even when all the particles are identical. Further, in counting the number of complexions (microstates) for deriving Maxwe11-Boltzmann statistics one usually assumes that interchange of two identical particles in two cells would give rise to a different microstate, since each particle can be tagged. The real reason for the appearance of $\mathrm{N}$ ! lies in the quantum mechanical concept of a "state" and the "indistinguishability" of identical particles, (for details see [31] p. 72).

In actual evaluation of the integral (3.4), one integrates first over the momentum coordinates and gets pf due to the kinetic energy by assuming point particles. Thas (3.4) gives

where

$$
Z_{\text {system }}=\frac{1}{N ! \Lambda^{3 N}} \int \exp -(U / k T) d \vec{q}
$$

$\Lambda=\left(h^{2} / 2 \pi m k T\right)^{\frac{1}{2}}$

The "hard core" is therefore included in the potential $\mathrm{U}(\overrightarrow{\mathrm{q}})$ of the system and consequently one can avoid divergence difficulties only by taking this into account in the integration of (3.7). For a system of charged particles this integral would diverge if we do not include the repulsive potential as in the original DH theory. But, contrary to the remark of Bogoliubov [4] mentioned before (see p.4 ), it would make this integral meaningful if we take this hard core into account while integrating (3.7).

The central problem of statistical mehcanics (both equilibrium and non-equilibrium) is to calculate the average potential of the system when the mutually interacting potential between two particles is known. Consequently, all the efforts 
in the past had been directed to evaluate the configuration integral

$$
\mathrm{Q}=\frac{1}{\mathrm{~N} !} \int \exp -(\mathrm{U} / \mathrm{kT}) \mathrm{d} \overrightarrow{\mathrm{q}}
$$

It should be noted that in the new approach one calculates the average potential by solving the pertinent nonlinear pde without making any assumption about the legitimacy of a sum of pair-wise interaction giving the exact or the average potential of the system. And once one gets this average potential, one can also use the conventional approaches . For example, one gets directly the pairdistribution function necessary for calculating the thermodynamic properties, without making any assumption about the superposition approximation of the distribution function theory. Also one does not need the cluster expansion scheme. But one must always remember that the volume available to a particle is not $\mathrm{V}$ but $[\mathrm{V}-(\mathrm{N}-1) \mathrm{b}]$. Mayer's clusters are point sets and in the evaluation of the cluster integrals one does not take into account that a cluster of actual particles has definite exclusion volume and the entire volume $V$ is not available to a particular cluster. Consequently, although it is obvious that an equation of state exists for any liquid or a solid, the usual cluster method as well as the formalism of the distribution function theory gives divergent virial expressions for high densities.

\section{THE NEW APPROACH}

\section{(i) Basic Concepts and Techniques.}

The starting point of this new approach is the same as in all other accepted methods, namely, to find the number of ways in which a given energy state of the macrosystem can be achieved. The specific techniques used here, however, are entirely different from conventional methods. This new approach does not suffer from the difficulties encountered in the original Boltzmann - Planck method for Interacting systems. It also circumvents the difficulties which confronted Fowler [38] due to the fact that only for a very few simple systems one could calculate the energy states and their degrees of degeneracy. These practical 
advantages are entirely due to the computational techniques employed in this new approach which enable one to calculate reliable results for both equilibrium and non-equilibrium phenomena.

The computational techniques are based on the following three theoretically we11 established principles.

(a) The phase space ( $\mu$-space) is partitioned into configuration space and momentum space. The justification of this from practical standpoint is also obvious from the well known fact that for classical systems all thermodynamic properties of the system due to the kinetic energy can be expressed separately from those due to the potential energy which gives the so-called "excess" functions.

(b) Due to finite sizes of particles, the physical space is divided into elementary non-overlapping cells of volume $b$, the exclusion volume of Boltzmann, The particles are then distributed amongst these cells by considering them as "image points" with the obvious restriction that a cell can either remaln vacant or is occupied by one particle on1y.

(c) Once we have determined the number of distinct ways in which the particles can be distributed in the physical space alone, the pf of the entire system could be easily calculated by utilizing the following two recipes:

$(\alpha)$ Since the number of cells in the micro-canonical layer $E_{i}$ and $E_{i}+\mathrm{dE}_{i}$ is $\left[2 \pi(2 m)^{3 / 2} E_{i}^{\frac{1}{2}} d_{i}\right] /\left(h^{3} / b\right)$ and for classical systems this number can be taken as a continuous function of energy, one can directly calculate the pf, $z_{k i n}$, due to kinetic energies of the particles alone. Thus, for a system of $\mathrm{N}$ particles, (with 3 degrees of freedom), we get

$$
\begin{aligned}
z_{\text {kin }} & =\text { w. } \sum_{s=1}^{N} \exp -\left(E_{i 1}+E_{i 2}+\ldots+E_{i N}\right) / k T \\
& =\text { w. }\left(b / \Lambda^{3}\right)^{N}
\end{aligned}
$$

where $\sum_{s=1}^{N} E_{i s}=E_{i}$;

$E_{i s}$ is the kinetic energy of the sth particle, $W$ is the number of ways in which $N$ 
particles can be distributed independently in the physical space to give the fixed kinetic energy $E_{1}$ of the given system and

$$
\Lambda=\mathrm{h} /(2 \pi \mathrm{mkT})^{\frac{1}{2}}
$$

It should be noted that the finite size of the particles directly enters into the pf. This procedure is further justified by the identical results which can be obtained for all thermodynamic properties for ideal gases by making the approximation $\mathrm{Nb} / \mathrm{V} \rightarrow \mathrm{o}$, as well as due to hard spheres using a more cumbrous but generally accepted method used by Dutta [14]. Dutta calculated the total thermodynamic probability $W^{\text {total }}$ as a product of the distribution in the configuration space and that in the momentum space. Thermodynamic properties were then obtained by maximizing $\mathrm{W}^{\text {total }}$ and connecting the entropy $\mathrm{S}$ for the equilibrium state by using Boltzmann's hypothesis $s=k \ell n w_{\max }^{\text {total }}$. Dutta did not consider non-equilibrium statistical mechanics.

(B) The most difficult problem of statistical mechanics is to calculate the average potential energy of the system. All the standard methods fail to evaluate the configuration integral correctly, as has been noted before. In this method one calculates the average potential by solving the pertinent nonlinear pde. For this It is enough to know the mutual potential energy of the two particles and one does not have to bother directly about many particle interactions.

Once we know this average potential, there are several ways in which one can calculate excess thermodynamic functions due to the potential field alone. The method used here is to utilize Debye-Hueckel's concept of ion-atmosphere and their technique to calculate the free energy of the system due to the potential field. It might be noted here again that though the original $\mathrm{DH}$ theory cannot be accepted even as a limiting theory, the concept of ion-atmosphere remains valid generally, (cf. Bagchi [7], also see section 5). This, of course, presupposes, (almost always fulfilled practically, cf. Onsager [50]), that the actual field prevailing in the system has on the average a potential. 
In order to carry out this programme we also need the distribution function for the number of particles around a "central" particle in the potential field alone. As shown before (see also below), this can be obtained from the number of ways in which the particles can be distributed in the physical space, if we subdivide it into thin layers within which the potential remains practically constant.

For the convenience of readers, concrete fllustrations are presented here briefly to show how this method works in practice. Two cases, namely, hard sphere system and fused alkali halides are treated here. The case of fused alkali halides has been chosen as a concrete real system for several reasons. First, the pertinent pde, namely, the modified PB equation, had been solved analytically and it gave excellent results for the nonlinear ion-atmosphere potential, (cf. Bagchi \& Plischke [24]). Further, even in the linear approximation this new method yielded reliable results for thermodynamic and transport properties of such systems, (see Table I), whereas the problem of charged particles cannot be tackled at all with mathematical consistency, if one follows the conventional methods. Secondly, one can use modified $\mathrm{PB}$ equation with sufficient approximation, since polarisation forces contribute between 2 to $3 \%$ of the total electrostatic energy of such systems and they have also been taken into account indirectly by the macroscopic dielectric constant. It should be also noted that short range forces are incorporated as exclusion volumes directly into the distribution function itself. Thirdly, the system does not suffer from complications encountered in solutions of strong electrolytes mainly due to the presence of water molecules. Finally, the most important reason is to convince the sceptics that it offers a direct a posteriori verification of the general validity of the Ion-atmosphere concept and of the actual "Debye Model". The theoretical proof given before, (cf. Bagchi [7]), is discussed again in section 5 .

Other cases involving mutually interacting potentials of the type $\psi(r)_{n} \equiv \sum_{-\infty}^{\infty} c_{n} r^{n},\left(c_{n}=\right.$ constant $)$, will be treated in a later publication. 
(ii) Hard Sphere System.

The system consists of $\mathrm{N}$ identical, non-penetrating particles with a given total energy $E$, the kinetic energy of the system, contained within the volume $V$ at a fixed absolute temperature $T$.

Let $\mathrm{b}$ be the exclusion volume associated with each particle. As pointed out rightly by Brillouin [51], the shape of the cell does not matter as long as the volume $\mathrm{V}$ is very large compared to $\mathrm{b}$. The number of cells in the volume is therefore given by

$$
\mathrm{M}=\mathrm{V} / \mathrm{b}
$$

The number of distinct ways in which $\mathrm{N}$ particles can be distributed amongst these $M$ cells such that a cell can either remain vacant or occupied by a single particle is

$$
\mathrm{W}=\frac{\mathrm{M} !}{\mathrm{N} !(\mathrm{M}-\mathrm{N}) !}
$$

The pf. of the system, in thi's case due only to the kinetic energies $E_{i s}$ of the individual particles, is therefore, (cf. eq. 4.2),

$$
z=W \cdot\left(b / \Lambda^{3}\right)^{N}
$$

Hence from (4.7) one can derive all the equilibrium thermodynamic properties of the system. Thus, using Stirling's approximation, we get

(a)

$$
\begin{aligned}
\ln \mathrm{Z}=\mathrm{N}\{\ln \mathrm{V} & +\frac{3}{2} \ln \left(\frac{2 \pi \mathrm{mkT}}{\mathrm{h}^{2}}\right)-\ln \mathrm{N} \\
& +(1-\mathrm{V} / \mathrm{Nb}) \ln (1-\mathrm{Nb} / \mathrm{V})\}
\end{aligned}
$$

(b) The energy of the system is

$$
\mathrm{E}=\mathrm{kT}^{2}(\partial \ln \mathrm{Z} / \partial \mathrm{T})_{\mathrm{V}, \mathrm{N}}=\frac{3}{2} \mathrm{NkT}
$$

(c) Specific heat at constant volume

$$
\mathrm{C}_{\mathrm{v}}=(\partial \mathrm{E} / \partial \mathrm{T})_{\mathrm{V}, \mathrm{N}}=\frac{3}{2} \mathrm{~N} \mathrm{k}
$$

Both $E$ and $C_{V}$ are the same as for perfect gases. 
(d) Entropy :

$$
\begin{aligned}
& S= \frac{E}{T}+k \ln Z \\
&=N k\left\{\frac{3}{2}+\frac{3}{2} \ln \left(\frac{2 \pi m k T}{h^{2}}\right)-\ln N+\ln V\right. \\
&\quad+(1-V / N b) \ln (1-N b / V)\}
\end{aligned}
$$

(e) Helmholtz free energy:

$$
\begin{aligned}
F(N, V, T)=-k T & \ln Z \\
=-N k T\{ & \frac{3}{2} \ln \left(\frac{2 \pi m k T}{h^{2}}\right)-\operatorname{lnN}+\operatorname{lnV} \\
& +(1-\mathrm{V} / \mathrm{Nb}) \ln (1-\mathrm{Nb} / \mathrm{V})\}
\end{aligned}
$$

All these relations give the well known expressions for ideal gases for $\mathrm{Nb} / \mathrm{V} \rightarrow 0$

(f) The virial expression :

As we have pointed out before, whatever be the actual value of $b$, it remains constant for a given system. Hence, from (4.12) we get for pressure p the expression

$$
p=-(\partial F / \partial V)_{T}=-\frac{k T}{b} \ln (1-N b / V)
$$

Equation (4.13) is known as Saha-Bose equation of state, (see Saha \& Bose [52], also Saha \& Srivastava [53]).

The usual form of the virial expression is obtained by expanding the logarithmic term in $(4.13)$.

$$
\frac{p V}{N k T}=1+b p+\frac{1}{3} b^{2} p^{2}+\ldots
$$

where $\rho \equiv \frac{\mathrm{N}}{\mathrm{V}}$ denotes the number density.

Since for physical reasons $\mathrm{Nb} / \mathrm{V}$ is always less than unity, the expressions (4.13 and 4.14) are always convergent for any actual density encountered in nature. This cannot be asserted for the conventional form of the virial expression for hard spheres. As proved before, (see Bagchi [1]), if one takes the values of virial coefficients given in the literature for hard sphere systems, the virlal serles diverges for high densities comparable to those of liquid argon near the 
triple point. Such discrepancies are caused by the fact that in the integration processes one had not taken into consideration the actual volume accessible to a particle. Further, it has been shown there that the volume $v$ available to a particle, if one follows Boltzmann and takes the virial coefficients as reported in the literature to be correct, one comes to the absurd physical result, namely, this available volume $v$ becomes negative. One can also see that for dilute gases, for which the calculation of Boltzmann were intended, these inconsistencles do not arise in practice. Nevertheless, in principle one cannot accept the given virial coefficients as correct. For details see Bagchi [1].

It might be noted that this method gives consistent results also for a binary mixture of hard spheres. All the expressions go over to the well known results for mixture of Ideal gases for $\mathrm{Nb} / \mathrm{v} \rightarrow 0$, (see Bagchi [2]).

(iii) Fused Alkali Halides.

The system contains $\mathrm{N}^{+}$positive ions and $\mathrm{N}^{-}$negative ions in a given volume $\mathrm{V}$ at the temperature T. The lons are completely dissociated. They are considered here as rigid spherical particles of radil $a_{+}$and $a_{-}$respectively. This model is supported also by the electron density distribution of their crystalline structures, (see ref. [16], p. 469). Their masses are $\mathrm{m}_{+}$and $\mathrm{m}_{-}$respectively.

Our first problem is to calculate the exclusion volumes $b_{ \pm}$of the two ions and to distribute the two lons amongst the cells of the physical space which would also permit us to calcualte the distribution of the two ions around a "central" Ion.

The $\mathrm{pf}, \mathrm{z}_{\mathrm{kin}}$, due to their kinetic energies is given by

$$
\mathrm{z}_{\text {kin }}=\mathrm{W} \cdot\left(\mathrm{b}_{+} / \Lambda_{+}\right)^{\mathrm{N}^{+}} \cdot\left(\mathrm{b}_{-} / \Lambda_{-}\right)^{\mathrm{N}^{-}}
$$

where $W$ represents the number of ways in which the ions can be distributed in the volume $V$ and $\Lambda_{ \pm}=\left(h^{2} / 2 \pi m_{ \pm} k T\right)^{\frac{1}{2}}$.

(a) Calculation of the cell volumes.

For a system containing only one kind of particles one can write down the correct expression for the exclusion volume $b$ as 


$$
b=\gamma b_{0} ; \quad b_{0}=\frac{4 \pi}{3} \sigma^{3} ;
$$

where $\gamma$ is the overlap correction factor and $\sigma$ is the diameter of the particle. But it is extremely difficult to calculate exactly the value of $b$ due to multiple overlapping of first nelghbours. For 1-1 salt with different lonic radil one would expect to use different exclusion volumes $b_{+}$and $b_{-}$for the two lons.

It should be noted here that it is not physically meaningful in such systems to take three exclusion volumes, $b_{+}, b_{-}$and $b_{+-}$, where $b_{ \pm}$represents the exclusion volume corresponding to the closest distance of approach of like lons and $b_{+-}$that due to unlike Ions, as was implied in the original paper of Dutta \& Bagchi [19]. This is primarily due to the fact that there is always a number of lons of opposite charges in between two 11ke ions. Further, later investigations by the author [23] using the exact nonlinear solution of the modified PB equation showed that in Ionic solutions where there is always at least a layer of water molecules between unlike 1ons, even a plausible exclusion volume $b_{ \pm}$would give physically inconsistent results for high concentrations and large lonic radil. These inconsistencles persist also in the case of fused salts. Also for other reasons noted below, practically the most useful and also theoretically more justiflable method is to take the same exclusion volume for the two ions, namely,

$$
b_{+-}=b_{-+}=b=r b_{0} ; b_{0}=\frac{4 \pi}{3}\left(a_{+}+a_{-}\right)^{3}
$$

The difficulty of calculating $\gamma$ exactly would still persist. The value of $\gamma$ calculated by using Boltzmann's model for collision cross-section in which the first neighbours (oppositely charged ions) are considered as points, (see Bagchi [3]), proved to be good enough to give rellable results for both equilibrium and nonequilibrium properties of fused alkali halides. Furth er, as shown below, if we take different values of $b_{+-}$and $b_{-+}$and apparently the most plausible method of distributing the lons, namely, the hypergeometric distribution, (cf. Feller [54]), 
we shall be confronted with intractable problems.

b) The calculation of $\mathrm{W}$ and the distribution of lons around a "central" Ion.

At first sight, it seems that the most satisfactory way of calculating $W$, the number of Independent ways in which the lons can be distributed in the physical space, is to modify the hypergeometric distribution of mathematical statistics and to follow the procedure given by Dutta \& Bagchi [19] but with only two exclusion volumes, one for each ion, instead of three $b_{+}, b_{-}, b_{+_{-}}$introduced in [19].

For distributing the lons we subdivide the volume $\nabla$ into thin layers of volumes $\nabla_{1}, \nabla_{2}, \ldots, \nabla_{m}$ in which the potential of the system $\psi_{1}, \psi_{2}, \ldots \psi_{m}$ remains constant. Let $\mathrm{N}_{1}^{ \pm}, \mathrm{N}_{2}^{ \pm}, \ldots, \mathrm{N}_{\mathrm{m}}^{ \pm}$denote the number of positive and negative Ions in these layers.

Now, let us first distribute the positive fons in cells of volume $b_{4}$. The number of such cells is $\nabla_{m} / b_{+}$. We distribute afterwards the negative lons into cells of volume $b_{-}$in the avallabie space $\left(V_{m}-N_{m}^{+} b_{t}\right)$. Then,

$$
w_{t-}=\frac{\mathbb{R}}{m} \frac{\left(\nabla_{m / b_{+}}\right) !}{N_{m}^{+} !\left(\frac{\nabla_{m}}{b_{t}}-N_{m}^{+}\right) !} \cdot \frac{\left(\frac{v_{m}-N_{m} b_{t}}{b_{-}}\right) !}{N_{m} !\left(\frac{v_{m}-N_{m}^{+} b_{t}}{b_{-}}-N_{m}^{-}\right) !}
$$

But if we distribute the negative lons first and afterwards the positive lons in the avallable space, we get

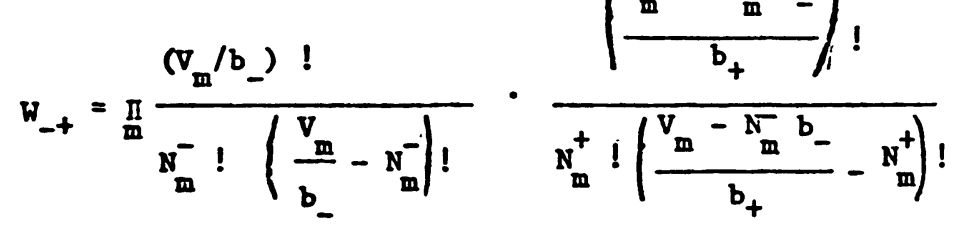


These two expressions are generally different. But the thermodynamic probability should be the same for the actual system, Independent of the way in which, for the sake of convenience, we have distributed the ions. If we recall that thermodynamic properties depend on the logarithm of $\mathrm{W}$, we can eliminate this assymetry originating from our particular arbitrary mode of description by the symetrization technique. Consequently, the correct "configurational thermodynamic probability" $\mathrm{W}$ is given by

$$
\ln \mathrm{W}^{=\frac{1}{2}}\left(\ln \mathrm{W}_{+-}+\ln \mathrm{W}_{-+}\right)
$$

Now, maximizing this subject to the following conditions

$$
\begin{aligned}
& \sum_{m} N_{m}^{+}=N^{+} ; \quad \sum_{m} N_{m}^{-}=N^{-} \\
& \sum_{m} N_{m}^{+} e_{+} \psi_{m}+\sum_{m} N_{m}^{-} e_{-} \psi_{m}=v,
\end{aligned}
$$

where $U$ is the total potential energy of the system and $e_{+}$are the charges of the ions, we get, (after omitting the subscripts), the distribution of the fons in each layer around a central ion the expressions

$$
\left.\begin{array}{l}
\frac{1}{n^{+} b_{+}}\left(1-n^{+} b_{+}\right) \frac{b_{-}-b_{+}}{2 b_{-}} \cdot\left(1-n^{-} b_{-}-n^{+} b_{+}\right) \frac{b_{-}+b_{+}}{2 b_{-}}=\exp \left(\nu_{+}+\mu e_{+} \psi\right) \\
\frac{1}{n^{-} b_{-}}\left(1-n^{-} b_{-}\right) \frac{b_{+}^{-} b_{-}}{2 b_{+}} \cdot\left(1-n^{-} b_{-}-n^{+} b_{+}\right) \frac{b_{+}^{+} b_{-}}{2 b_{+}}=\exp \left(\nu_{-}+\mu e_{-} \psi\right)
\end{array}\right\}
$$

$\nu_{ \pm}, \mu$ are the Lagrangian parameters of the variation process, which can be calculated in the usual way, (see below).

Although in principle $n^{ \pm}(r)$, the number of lons at a distance $r$ from the central ion, can be expressed in terms of the potential $\psi$, in practice for further calculations the expressions $(4.23)$ become intractable. Further, they show some internal physical inconsistencies, just like the original distribution of Dutta \& Bagchi [19]. For example, if we take $b_{+}=b_{-}=b$, (say), $w$ becomes the same as the usual hypergeometric distribution of two kinds of particles distributed in identical boxes. In this case, $(4.23)$ become 


$$
\begin{aligned}
n^{+} b & =\frac{1-n^{-} b}{\exp \left(\nu_{+}+\mu e_{+} \psi\right)+1} \\
n^{-} b & =\frac{1-n^{+} b}{\exp \left(\nu_{+}+\mu e_{-} \psi\right)+1}
\end{aligned}
$$

However, in this case $X$, the inverse of the radius of the 1on-atmosphere, becomes independent of the exclusion volume $b$ and has the value $x_{D}^{2}=\frac{8 \pi \varepsilon^{2}}{D k T} n_{0}$ for $1-1$ salt, the same expression encountered in the original $\mathrm{DH}$ theory, $\mathrm{n}_{0} \frac{\mathrm{d}}{\mathrm{den}} \mathrm{tes}$ the macroscopic average number density of the ions.). Consequently, these distribution functions (4.23) and 4.24) cannot be accepted as the correct distributions of lons. It should be noted that for fused KF, the exclusion volumes of the lons have almost the same value.

It should also be noted that if $\mathrm{n}^{-} \mathrm{b} \ll 1, \mathrm{n}^{+} \mathrm{b} \ll 1$, which however 1 s not permissible elther for concentrated solutions or for fused salts, we get the distribution functions

$$
\begin{aligned}
& \mathrm{n}^{+}(\mathrm{r})=\frac{1}{\mathrm{~b}} \frac{1}{\exp \left(\nu_{+}+\mu \mathrm{e}_{+} \psi\right)+1} \\
& \mathrm{n}^{-}(\mathrm{r})=\frac{1}{\mathrm{~b}} \frac{1}{\exp \left(\nu_{-}+\mu e_{-} \psi\right)+1}
\end{aligned}
$$

It is curious that these expressions (4.25) which can be obtained by nonpermissible approximations both from the or $I_{g}$ inal Dutta \& Bagchi distribution function and from the intractable epxressions (4.23) give quite satisfactory results even in the linear approximation of the Ion-atmosphere potentials both for solutions of strong electrolytes as well as for fused alkall halldes. The success of this distribution for calculating both equilibrium and non-equilibrium properties of charged systems lies essentially in the fact that here $x$ depends explicitly on the exclusion volume b. For example, for 1-1 salt, we get

$$
x^{2}=x_{D}^{2}\left(1-n_{0} b\right)
$$


It might be noted that the same formula (4.25) was derived by Wicke \& Eigen [22] from considerations of chemical equilibrium and the law of Mass Action between sites occupied by hydrated fons and vacant sites. Obviously, their mode of derivation cannot be considered theoretically as satisfactory. Consequently, presumably impressed by the practical success of (4.25), Falkenhagen, (1953, ref [21]) derived a distribution function from the original Boltzmann distribution by correcting it formally for the finite sizes of the ions. But the distribution function derived by him also suffers from the inconsistency that for 1-1 salt $x$ obtained from this distribution becumes identical with Debye's $X_{D}$. Hence, this distribution function is also not physically acceptable.

Consequently, Bagchi [3] derived the expression (4.25) from Boltzmann model of the exclusion volume. The mode of derivation given there is physically and mathematically consistent. One can derive similar distribution functions rigorously provided the particles are distributed in their respective cells independently, (see below). Extensive calculations by Bagchi [23] for solutions of strong electrolytes with the exact nonlinear Ion-atmospheric potential as well as the results obtained for equilibrium and transport properties of fused alkali halides leave no doubt that physically the most satisfactory distribution function of the Ions around a central ion is given by

$$
\mathrm{n}^{ \pm}(\mathrm{r}) \mathrm{b}_{ \pm}=\frac{1}{\left.\exp \mid \nu_{ \pm}+\mu \mathrm{e}_{ \pm} \psi\right)+1}
$$

From thermodynamic relation $(\partial S / \partial E)_{V}=\frac{1}{T}$ and Boltzmann's relation $S=k \ell n W_{\max }$, one gets $\mu=1 / \mathrm{kT}$. The Lagrangian parameters $\nu_{ \pm}$are obtained from the boundary condition $\psi \rightarrow 0$ as $\mathbf{r} \rightarrow \infty$. Thus,

$$
A_{ \pm} \equiv \exp \left(\nu_{ \pm}\right)=\frac{1}{n_{0} b_{ \pm}}-1 ; \text { for } 1-1 \text { salt }
$$

Substituting these constants in (4.27) one can easily see that for $b \rightarrow 0$, one gets 
the usual Boltzmann distribution

$$
n^{ \pm}(r)=n_{0}^{ \pm} \exp \left(-e_{ \pm} \psi / k T\right)
$$

Theoretically, the only consistent method of deriving the distribution function ( 4.27$)$ is to calculate the "configurational thermodynamic probability" W by distributing the fons independently into their respective cells. That is,

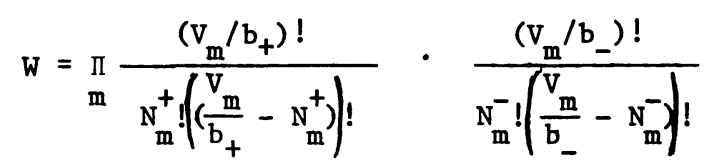

The essential feature is thus to distribute the ions in their cells in the total volume $\mathrm{V}$ independently. In fact, this procedure is adopted to obtain the correct entropy of a mixture of gases. Also, Sommerfeld in his book "Thermodynamics \& Statististics"uses this method. He distributes the particles of the two gases independently in their own $\mu$-spaces. Physically, this can be justified as follows.

By blocking the sites, the volume available to the particles will be less than $\mathrm{V}$ and consequent1y, we would be unwarrantedly decreasing the number of microstates. It must be remembered that the total number of microstates can be obtained only when all the cells of the volume $V$ are available to each particle, since in some microstates any particle can occupy any cell of the entire physical space. Finally, it should be recalled that the justification of any physical statistics for any particular situation rests on a posteriori verification. Consequently, one can accept (4.27) or its simpler version (4.25) as a correct description of the statistics under consideration. At least, physical results, for both thermodynamic and transport properties, calculated from these distribution functions proved beyond doubt that they are adequate enough to treat satisfactorily statistical mechanics of fused salts.

(c) Calculation of the free energy of the system due to the potential field. First, we calcualte the potential around a central ion from the modified PB 
equation for the given distribution function. For example, from (4.27) we get for a binary salt

$$
\nabla^{2} \psi=-\frac{4 \pi}{D} \rho=-\frac{4 \pi}{D}\left[z_{+} \varepsilon n^{+}(r)+z_{-} \varepsilon n^{-}(r)\right]
$$

or,

$$
\begin{aligned}
\nabla^{2} \lambda & =f(\lambda) \\
& =-\frac{4 \pi \varepsilon^{2}}{D k T}\left\{\frac{z_{+}}{b_{+}\left[A_{+} \exp \left(Z_{+} \lambda\right)+1\right]}+\frac{z_{-}}{b_{-}\left[A_{-} \exp \left(z_{-} \lambda\right)+1\right]}\right\}
\end{aligned}
$$

and

$$
\begin{aligned}
x^{2} \equiv \dot{f}^{\prime}(0) & =-\frac{4 \pi \varepsilon^{2}}{D k T} z_{+}{ }_{-}{l_{n}^{+}}_{0}^{+}\left(1-n_{0}^{-} b_{-}\right)+n_{0}^{-}\left(1-n_{0}^{+} b_{f}\right\}^{\}} \\
& =\frac{8 \pi \varepsilon^{2}}{D k T} n_{0}\left(1-n_{0} b\right) ; \quad\left(\text { for } 1-1 \text { salt and } b_{+}=b_{-}=b\right), \\
& =x_{D}^{2}\left(1-n_{0} b\right)
\end{aligned}
$$

Here, $\lambda=\varepsilon \psi / \mathrm{kT} ; \varepsilon=$ magnitude of the elementary charge; $z_{ \pm}=$the valencles of the 1ons; $D=$ macroscopic dielectric constant. $A_{ \pm} \equiv \exp \left(v_{ \pm}\right)$

The nonlinear equation of Poisson-Boltzmann type given by (4.31) had already been solved and gave excellent results for the average potential of the system, (cf. ref. $[24]$ ). Let $\psi(r)$ be this potential. At the surface of the central ion of charge $z_{+} \varepsilon$ and radius $a_{+}$, the potential due to the atmospheric ions is

$$
\phi\left(a_{+}\right)=\psi\left(a_{+}\right)-\frac{z_{+} \varepsilon}{D a_{+}}
$$

For the negative central ion, the potentials $\psi$ and $\phi$ would have just the opposite sign.

Consequently, following the technique used by Debye \& Hueckel [10], one can 
calculate $f_{1}$ el $(N, V, T)$, the free energy of the particular system in which the Ion " 1 " is the central 1on. It should be noted that this is the free energy due to the central Ion as well as all other atmospheric fons of the subsystem in which ' 1 ' plays the role of the central ion. For example, for a positive central 10n, we get the expression (4.36) for the free energy.

$$
f_{+}^{e l}=\int_{0}^{1} \phi\left(\nu z_{+} \varepsilon, a_{+}\right) z_{+} \varepsilon d \nu
$$

Hence, the free energy of the given system due to the potential field alone is given by

$$
F^{e l}(N, V, T)=N_{+} f_{+}^{e l}+N_{-} f_{-}^{e l}
$$

For the linear approximation of the lon-atmosphere potential, the free energy $f_{1}^{e 1}(N, V, T)$ of the subsystem In which "I" Is the central ion we get the well known expression

$$
f_{1}^{e 1}=\frac{z_{1}^{2} \varepsilon^{2}}{D a_{1}} \Gamma\left(x a_{1}\right)-\frac{z_{1}^{2} \varepsilon^{2}}{2 D a_{1}}
$$

where $\Gamma(x a)=\frac{1}{(x a)^{2}}\left[x^{a}-\ln (1+x a)\right]$

The electrostatic free energy due to the nonlinear potentlal had already been calculated for solutions of strong electrolytes, (cf. Bagch1 [23]). The corresponding values for fused alkall halides are being calculated now. All these reaults will be published in a separate paper.

Table I gives some thermodynamic properties as well as the electric specific conductivities of a few fused alkali halldes in the linear approximation of the Ion-atmosphere potential.

In view of the fact that it is extremely difficult to determine the exact value of $b$ the results appear to be quite satisfactory. The large discrepancles for the values of compressibility and expansivity are probably to be ascribed to the existence of micro-clusters in the liquid state, (cf. ref. [15], which had not yet been taken into account in the formalism of this new approach. The nonlinear 
Table I. Thermodynamic and Transport Properties of Fused Alkali Halides

\begin{tabular}{|c|c|c|c|c|c|c|c|c|c|c|}
\hline \multirow[t]{2}{*}{ Salt } & \multicolumn{2}{|c|}{$\begin{array}{c}\text { Entropy } \\
\text { (cal/mole.k) }\end{array}$} & \multicolumn{2}{|c|}{$\begin{array}{l}\text { Specific Heat } \\
\text { at const. press. } \\
\text { (cal/mole. R) }\end{array}$} & \multicolumn{2}{|c|}{$\begin{array}{l}\text { Isothermal } \\
\text { compressibility } \\
\times 10^{12} \\
\left(\mathrm{~cm}^{2} / \text { dyne) }\right.\end{array}$} & \multicolumn{2}{|c|}{$\begin{array}{l}\text { Expansivity } \\
\text { per }{ }^{\circ} \mathrm{C} \\
\times 10^{4}\end{array}$} & \multicolumn{2}{|c|}{$\begin{array}{l}\text { Specific } \\
\text { conductivity } \\
\text { ( }(\Omega . \mathrm{cm})^{-1}\end{array}$} \\
\hline & Calc. & Expt. & Calc. & Expt. & Calc. & Expt. & Calc. & Expt. & Calc. & Expt \\
\hline LiF & 28.39 & 30.6 & 18.34 & 15.5 & 10.99 & - & 1.47 & 2.71 & 13.01 & 8.66 \\
\hline $\mathrm{NaF}$ & 37.79 & 37.4 & 15.76 & 16.4 & 19.14 & - & 1.68 & 2.89 & 5.34 & 4.95 \\
\hline $\mathbf{R F}$ & 41.32 & 39.2 & 14.76 & 16.0 & 24.18 & - & 1.75 & 3.41 & 4.04 & 3.60 \\
\hline LiCl & 31.30 & 33.5 & 19.44 & 15.3 & 33.74 & 19.2 & 2.14 & 2.87 & 7.06 & 5.67 \\
\hline $\mathrm{NaCl}$ & 41.85 & 40.8 & 15.65 & 16.0 & 43.5 & 28.7 & 2.23 & 3.07 & 3.78 & 3.58 \\
\hline $\mathrm{RCl}$ & 45.93 & 42.2 & 14.12 & 16.0 & 46.42 & 36.7 & 2.12 & 3.58 & 2.62 & 2.24 \\
\hline LIBI & 34.71 & 34.4 & 19.36 & 15.6 & 49.04 & 21.4 & 2.54 & 2.59 & 5.96 & 4.73 \\
\hline $\mathrm{NaBr}$ & 45.14 & 42.4 & 15.51 & 16.7 & 57.37 & 31.5 & 2.51 & 3.50 & 2.99 & 2.90 \\
\hline $\mathbf{R B I}$ & 49.16 & 45.6 & 13.91 & 16.7 & 57.91 & 39.5 & 2.30 & 3.91 & 2.09 & 1.61 \\
\hline
\end{tabular}

The parameters used in the calculation are given below.

The different values of each parameter correspond to the salts in their serial order as given in the table.

v $\left(\mathrm{cr}^{3}\right)=14.4 ; 21.6 ; 30.6 ; 28.3 ; 37.5 ; 48.8 ; 34.4 ; 44.1 ; 55.9$

$T(\mathrm{R})=1121 ; 1268 ; 1131 ; 883 ; 1073 ; 1043 ; 823 ; 1020 ; 1007$

D $\quad=1.74 ; 1.56 ; 1.64 ; 2.25 ; 2.03 ; 1.94 ; 2.56 ; 2.27 ; 2.12$

b $\left(\mathrm{cm}^{3}\right) \times 10^{24}=10.79 ; 15.66 ; 24.73 ; 19.61 ; 26.71 ; 39.33 ; 23.11 ; 30.99 ; 44.83$

$\Lambda_{+} \times 10^{10}=19.80 ; 10.23 ; 8.30 ; 22.31 ; 11.12 ; 8.65 ; 23.11 ; 11.40 ; 8.80$

$\Lambda_{-} \times 10^{10}=11.97 ; 11.25 ; 11.91 ; 9.87 ; 8.95 ; 9.08 ; 6.81 ; 6.12 ; 6.16$

$a_{+}(\mathrm{cm}) \times 10^{8}=0.68 ; 0.95 ; 1.33 ; 0.68 ; 0.95 ; 1.33 ; 0.68 ; 0.95 ; 1.33$

a $(\mathrm{cm}) \times 10^{8}=1.36 ; 1.36 ; 1.36 ; 1.81 ; 1.81 ; 1.81 ; 1.95 ; 1.95 ; 1.95$

$x^{-}\left(\mathrm{cm}^{-1}\right) \times 10^{-8}=7.01 ; 5.76 ; 4.78 ; 5.12 ; 4.20 ; 3.63 ; 4.55 ; 3.78 ; 3.31$

$A^{\prime}(x a) \times 10^{3}=1.64 ; 1.95 ; 2.11 ; 2.16 ; 2.65 ; 2.79 ; 2.50 ; 3.05 ; 3.12$

(The values have been calculated by Messers N. Mukerj! . and C. Papanastasopoulos) 
potentials are expected to give better results.

(d) Transport Properties of Fused Alkali Halides.

As already noted before, one cannot use the expansion scheme of Chapman \& Enskog for calculating transport properties for high densities. But it had been shown before by Falkenhagen, (1958, cf. [21]), that for concentrated solutions of strong electrolytes one could calculatesatisfactorily their transport properties, even in the linear approximation of the ion-atmosphere potential, if one used the distribution function (4.25). We shall show here that the theory worked out by Debye, Onsager, Falkenhagen and their co-workers for irreversible phenomena of electrolytic solutions for linear approximation also yields satisfactory results for fused alkali halides. As an illustration, the phenomenon of electric conductance is treated here. In a later paper other transport properties, like viscosity and diffusion, will be treated from the theory available for electrolytic solutions as well as from Boltzmann equation,

$$
\frac{\partial f}{\partial t}+\vec{v} \nabla_{r} f+\vec{a} \nabla_{v} f=(\partial f / \partial t)_{\text {collision }},
$$

by taking into account the internal field due to the lon-atmosphere drag.

In the linear approximation, the expression for electric specific conductivity in ionic solutions is given by, (cf. Falkenhagen, 1958 ref. [21]),

$$
\lambda=\lambda_{\infty}-\mathbf{A} \lambda_{\infty}-\mathbf{B} \lambda_{\infty}
$$

A represents the effect of ion-atmospheric drag on the conductance at infinite dilution $\lambda_{\infty}$. The coefficient $B$ is due to the electrophoretic effect which we need not consider in the case of fused salts. For 1-1 salt, (see Falkenhagen 1958),

$$
A=\frac{\varepsilon^{2} \chi}{2 D k T} \cdot A^{\prime} ; A^{\prime}(x a)=\frac{1}{3}(2-\sqrt{ } 2) \frac{1}{(1+\chi a)\left(1+\frac{\sqrt{ } 2}{2} x a+\frac{1}{6} \chi^{2} a^{2}\right)}
$$

In case of solutions of electrolytes $\lambda_{\infty}$ is obtained from experimental results. In the case of fused salts we have to calculate it. 
Physically $\lambda_{\infty}$ means that in the calculation of the conductivity the effect of ionatmosphere does not play any role. In our model, the motion of a particle is determined by the effective field $\vec{E}_{\text {eff }}=\vec{E}_{-} \vec{E}_{\text {int }}$ and the collisions of hard spheres. Here $\mathrm{E}_{\text {int }}$ is the internal field due to the ion-atmosphere drag. Consequently, $\lambda_{\infty}$ is given by the well known expression

$$
\lambda_{\infty}=\sum_{1} n_{1} e_{1}^{2} \tau_{1}
$$

$\tau$ is the inverse of the collision frequency of hard spheres and is given by, (cf. Chapman \& Cowling [6] pp 87-88).

$$
\begin{aligned}
& \tau_{+}=\frac{n_{+}^{0}}{N_{++}+N_{+-}} \\
& \tau_{-}=\frac{n \underline{-}}{N_{--}+N_{+-}}
\end{aligned}
$$

where, for 1-1 salt,

$$
\begin{aligned}
& \mathrm{N}_{++}=4 \mathrm{n}_{0}^{2} \sigma_{+}^{2}\left(\pi \mathrm{kT} / \mathrm{m}_{+}\right)^{\frac{1}{2}} \\
& \mathrm{~N}_{--}=4 \mathrm{n}_{0}^{2} \sigma_{-}^{2}\left(\pi \mathrm{kT} / \mathrm{m}_{-}\right)^{\frac{1}{2}} \\
& \mathrm{~N}_{+-}=2 \mathrm{n}_{0}^{2} \sigma_{+-}^{2}\left[\frac{2 \pi \mathrm{kT}\left(\mathrm{m}_{+}+\mathrm{m}_{-}\right)}{\mathrm{m}_{+} \mathrm{m}_{-}}\right]^{\frac{1}{2}}
\end{aligned}
$$

We have to note here that the collision diameter of the hard spheres must be taken as those pertaining to the radius of the exclusion volume $b$ and not to the actual radil of the particles. Also we have to remember that in alka halides the closest distance of approach between the centres of unlike lons is $\sigma_{f_{-}}=2 a_{b}$ and that between like ions is $\sigma_{11}=2 \sqrt{2} a_{b}$, where $a_{b}$ is given by:

$$
b=\frac{4}{3} \pi a_{b}^{3}
$$

It is obvious that the striking success of this new approach, as had been pointed out before, is essentially due to the partition of $\mu$-space. The motion of a particle, governed by the effective potential field, is only hindered by the elastic collisions of hard spheres. The results show that transport properties even at high densities are determined by binary collisions only. This is also to be expected physically, since it is very unlikely that moving particles will suffer simultaneous many body collisions. 


\section{ION-ATMOSPHERE THEORY OF STRONG ELECTROLYTES}

The concept of ion-atmosphere around a central fon was introduced by Debye \& Huecke1 [10] to calculate in an ingenious way the "excess" free energy of a system of particles interacting with Coulomb forces. The Importance of their technique lies in the fact that a straightforward calculation of the configuration integral, which must include all types of forces and, in particular, short range forces, is not possible unless we modify the avallable techniques of evaluating this integral. And in view of the difficulties encountered with a much simpler system like hard spheres mentioned before, it is doubtful whether the calculation of such a complicated configuration integral would be feasible at all. The only practical and useful method, which moreover is physically and mathematically consistent, of treating such systems lies in utilizing this concept of ionatmosphere as in this new approach and in the modified DH theory. The DH technique should not be looked upon as a mathematical trick only. The existence of ion-atmosphere as a physical reality had been directly demonstrated by DebyeFalkenhagen effect and Wien effect in ionic solutions, (see Falkenhagen 1953, ref. [21]). Further, the general statistical validity of the ion-atmosphere concept had been proved theoretically before, (see Bagchi [7]). Moreover, the validity of this technique had been established a posteriori in the cases of fused salts and ionic solutions, (using the modified DH theory), for both equilIbrium and non-equilibrium phenomena.

It is rather strange that in view of the long established fact that the original DH theory is mathematically meaningless and inconsistent, (see Kramers [8], Gronwall [9], Halpern [55], Berlin \& Montroll [56], Bogoliubov [4], Bagchi [7]), it is still believed that the DH theory in which only the Coulomb forces are taken into account is strictly valid as a limiting theory. Instead of accepting the proven fact that the original $\mathrm{DH}$ theory cannot be accepted in 
principle even as a limiting theory, varlous authors have tried to improve the original DH theory by purely ad hoc schemes and recipes (see section 5.1x). The main objective of this section is therefore to remove the erroneous views persisting in the literature about the original $\mathrm{DH}$ theory and the ion-atmosphere concept as such.

Debye-Hueckel technique was scrutinised critically first by Fowler, ([57], see also [38]). Afterwards, Onsager [50] and Kirkwood [58] had discussed the foundation of this theory exhaustively. Nevertheless, as shown below, some important aspects of $\mathrm{DH}$ technique had been overlooked. As a result, it is now generally,(albeit erroneously), believed that the concept of ion-atmosphere and the use of $P B$ equation to determine the average electrostatic potential of the system are not self-consistent.

Let us then first dispel these erroneous notions by discussing the original DH theory.

(i) Debye-Hueckel Model.

In order to appraise the new point of view correctly, it is essential that one notes carefully the procedures actually pursued by Debye \& Hueckel to calculate the electrostatic free energy of the system.

For simplicity, let us consider a binary electrolytic solution consisting of completely dissociated $\mathrm{N}^{+}$positive and $\mathrm{N}^{-}$negative ions. Debye \& Hueckel did not treat directly the actually given system but considered this given system splitted into $\mathrm{N}\left(=\mathrm{N}^{+}+N^{-}\right)$independent part-systems in which each subsystem was characterized by a specific ion, say the ion " 1 ", as the central ion and all the remaining ( $\mathrm{N}-1$ ) ions were distributed in this particular subsystem in a characteristic way depending on the potential $\psi_{i}(r)$ of all the $N$ ions present in this subsystem. This potential was calculated from the PB equation in which the charge density at a distance $r$ from the central ion was given by Boltzmann's 
distribution formula. Now, from this $\psi_{i}\left(e_{i}, x, a_{i}\right)$, the potential at the surface of the central Ion of radius $a_{i}$, they determined the potential due to the atrospheric ions.

$$
\phi_{i}\left(e_{1}, x, a_{i}\right)=\psi_{1}\left(e_{i}, x, a_{i}\right)-\frac{e_{i}}{\operatorname{Da}_{i}}
$$

Here $D$ denotes the macroscopic dielectric constant of the medium. For the origial $\mathrm{DH}$ theory,

$$
x_{D}=\frac{4 \pi}{D K T} \underset{i=1}{N} \cdot n_{0}^{i} e_{i}^{2}
$$

Note that the usual physical interpreation of $x^{-1}$ as the radius of the ion-atmosphere is not physically meaningful, (see Table 1 and for detailed discussions the next paper). The free energy $f_{I}^{e l}(N, V, T)$ of this particular subsystem due to the potential field alone was then calculated by a "thought" process, namely, simultaneous charging up, (at first considered as uncharged); all the $\mathrm{N}$ ions in this subsystem at the same relative rate and keeping the spatial arrangement fixed. Thus,

$$
\begin{aligned}
f_{i}^{e l} & =f_{v=0}^{1} \phi_{i}\left[v e_{i} ; x\left(v e_{i}, \sum_{j} v e_{j}\right) ; a_{i}\right] e_{i}^{d v} \\
& =e_{i} p_{i}\left(x, a_{i}\right)
\end{aligned}
$$

Here [] denotes the argument of $\phi_{i}$ and () those of $x$ and $p_{i}$. Finally, the desired total electrostatic free energy of the given system was obtained from the relation (5.4).

$$
\mathrm{F}^{\mathrm{el}}=\mathrm{N}_{+} \mathrm{f}^{\mathrm{el}}+\mathrm{N}^{-} \mathrm{f}_{-}^{\mathrm{el}}
$$

It is obvious from (5.4) that in $\mathrm{DH}$ method one is treating the given systez not as one system but as an independent collection of $\mathrm{N}$ subsystems, each of which contains $(\mathrm{N}-1)$ ions of the given system around a particular central ion " $i " \psi_{i}(r)$ is the average potential of this particuiar subsystem. The electrostatic free energy of this subsystem is $e_{i} p_{i}\left(x, a_{i}\right)$, (cf. eq. 5.3). It is to be specially noted that this is the excess free energy not of the central ion " $i$ " but of this particular subsystem containing all the $\mathrm{N}$ ions of the given system. 
Once we recognize this basic fact and in view of (5.4) there can be no other logically consistent interpretation of $\mathrm{DH}$ technique, the fundamental criticism of Onsager [50] about the integrability condition for the electrostatic free energy of the system and consequently, against the validity of the ionatmosphere concept can be shown to be irrelevant. Before we prove this, let us first discuss briefly other pertinent criticisms raised against $\mathrm{DH}$ technique main1y by Fowler, (see [38], [57]).

(ii) Fluctuation Term.

Fowler's criticism against the use of Poisson equation does not refer to the actual electrostatic Coulomb potential. From electromagnetic theory, using the technique of Green's function, it can be easily proved that Poisson equation is valid for all potentials which vary as $1 / \mathrm{r}$, whether the system consists of discrete or continuous charge distributions. Kirkwood [58] had also shown that the use of Poisson equation was justified in the solution of strong electrolytes. Fowler's objection was rightly directed to the use of Poisson equation for the average potential $\bar{U}$. If Poisson equation was applicable for the actual potential $U$ of the system, he showed that $\overline{\mathrm{U}}$ satisfied the equation (5.5):

$$
\nabla^{2} \bar{U}+\frac{4 \pi}{D} \bar{\rho}=-\frac{1}{K T}\left\{\overline{(\nabla U)^{2}}-(\nabla \bar{U})^{2}\right\}
$$

Consequently, the function $\bar{U}$ does not satisfy Poisson's equation unless the fluctuation term, the right hand side of eq. (5.5), vanishes. Onsager [50] had shown that the fluctuation term was nearly cancelled by a supplementary term due to short range forces. The term cancels exactly for infinite distances of separation of the ions. Halpern [55] had noted that with increasing concentrations it would be more justified to neglect this term. According to Fowler [57], "criticism of this is possible but cannot be sustained so that within the limits imposed correct." Further, in his monograph [38] he concludes that "for the validity of the neglect of the fluctuation terms no general condition has yet 
been obtained. At present it seems possible only by verification a posteriori, p. 265". Results obtained from the modified DH theory as well as from this new approach for fused alkali halides have certainly verified this conclusion a posteriori. Anyway, it is now generally believed, I think rightly, though the exact proof is not available, that one can legitimately neglect this term for statistical mechanical considerations.

(iii) The Average Charge Density $\bar{\rho}$.

Kirkwood [58] had also shown that the exact expression for the average charge density may differ from the expression used in PB equation by an order of $1 / \mathrm{N}$. Consequently, this is of no consequence for macrosystems.

(iv) The Dielectric Constant D

The objection raised against the use of $(\overline{\rho / D})=\bar{\rho} / \bar{D}$ used in the theory is not relevant for the ion-atmosphere concept per ge. In fused alkali halides, for convenience of calculation and the use of modified PB equation, one takes the macroscopic dielectric constant to take into account indirectly the effect of polarisation forces. It is theoretically permissible and is also adequate enough since it is known that polarisation forces contribute only a very small portion to the total electrostatic energy of the system.

This objection is also not relevant for the (physically unrealistic) continuum model of the medium having a definite value of $D$. It is a legitimate and theoretically consistent approximation. Actually, the dielectric constant of the medium (water molecules) on the surface of the ion widely differs from the average macroscopic value. It can easily be shown from electromagnetic theory that the dielectric constant that matters is its value just outside the effective ionic radius. These technical difficulties can be overcome only by applying the theory, based on the same principle and technique, to the system composed of discrete.ions and discrete water molecules and by taking all types 
of forces into consideration. Of course, then we shall have to use a proper pde, instead of Poisson's equation. But this does not invalidate the concept of ion-atmosphere and the basic technique invented by Debye \& Hueckel.

(v) Onsager's Criticism About The Integrability Condition.

It is evident that the integrability condition should have to be applied to the total electrostatic free energy of the system and not to that of the subsystem.

The present position regarding this question can be summed up as follows, (cf. Fowler \& Guggenheim [59] ).

It was shown that in the linear approximation, DH solution was self-consistent, but this self-consistency was lost for unsymmetrical electrolytes and also if we took a few terms of the exact nonlinear solution. The argument that the linear approximation gives consistent results, whereas the complete solution does not, cannot have any force, since, as noted by Bagchi [18] and subsequently proved by SenGupta [60], in most cases where linearized DH theory is supposed to be valid, the linearity condition $e_{i} \psi_{i} / k T \ll 1$ is violated. For example, in the case of $\mathrm{NaCl}$ solution of $0.001 \mathrm{~m}$ SenGupta found $e_{i} \psi_{i} / \mathrm{kT}$ even at a distance of $4.4 \mathrm{~A}^{\circ}$ had the value 1.586. Moreover, if we note that the original $\mathrm{DH}$ theory cannot be accepted as a correct one, even when ionic exclusion volumes do not play any significant role and can safely be ignored, all these arguments about self-consistency of $\mathrm{DH}$ solution, linear or complete, are not adequate to lead to any rigorous or justifiable conclusion about the validity of the ion-atmosphere concept and $\mathrm{DH}$ technqiue.

Now, Onsager [50] came to the conclusion that $\mathrm{DH}$ technique gave inconsistent results about the integrability condition not merely from the facts mentioned above but from a far more general consideration. He argues, "if $\omega_{i j}(r)$ denotes the work (against the average force) in bringing two ions " $i$ " and " $j$ " 
from infinity to the distance $r$ one might expect $\omega_{1 j}(r)=\omega_{j 1}(r) "$. That is, in this case $e_{i} \psi_{j}(x)=e_{j} \psi_{i}(x)$. This argument is no doubt correct if $\psi(x)$ were potential of the system specifying the positions of all the lons in all configurations and treating the entire solution as the only one given system. But it loses its validity as soon as we note that in DH method of calculation of electrostatic free energy, the system is not treated as one system, but, as noted above, the given system is treated as $N$ independent subsystems. This follows not only from eqs. (5.3) and (5.4) but also from the well known fact that the total electrostatic energy of the system is given by

$$
u^{e l}=\frac{1}{2} \sum_{1=1}^{N} e_{1} \phi_{1}
$$

\section{(vi) General Proof of The Integrability Condition}

The proof becomes almost trivial if we note the actual Debye model used in DH technique. As noted above, the total electrostatic free energy of the system is given by, (cf. eq. 5.4),

$$
F^{e l}=f_{1}^{e l}+f_{j}^{e l}+\sum_{m=1}^{N-2} f_{m}^{e l}
$$

It is obvious that the integrability condition should be applied to this total $F^{e l}$ and not to individual $f_{m}^{e l}$, the free energy of the particular subsystem in which the fon " $\mathrm{m}$ " is the central ton. The integrability condition means that $\mathrm{dF}^{\mathrm{el}}$ should be a perfect differential. If we note that in each subsystem all the Ions of the given system are present, (the atmospheric ions are contained in the expression $x$ ), It would be wrong to assume that the criterion for the total differentiability is given by

$$
\frac{\partial p_{i}\left(x, a_{i}\right)}{\partial e_{j}}=\frac{\partial p_{j}\left(x, a_{j}\right)}{\partial e_{i}}
$$

The consistency requires that

$$
\frac{\partial^{2} F}{\partial e_{i} \partial e_{j}}=\frac{\partial^{2} F^{e l}}{\partial e_{j} \partial e_{i}}
$$


Since $F^{e l}$ as well as individual $f_{m}^{e l}$ 's are analytic functions of the parameters involved, the eq (5.9) is obviously satisfied.

(vi1) Debye-Heuckel's and Guentelberg's Methods.

From eq. (5.3) it is clear that $f_{i}{ }^{e l}$ represents the electric work of charging up all the ions simultaneously in the particular subsystem where ' 1 ' is the central ion. The activity coefficient $\gamma_{1}$ of the type of ion " $i$ " is then given by, (cf. [59]),

$$
\text { kT In } \gamma_{1}=\mu_{1}^{e l} ; \mu_{i}^{e l}=\frac{\partial F^{e l}}{\partial N_{i}}
$$

It is important to remember eq. (5.4) and to note that by this process we have taken into account automatically the fact that the fon " 1 " exists also in the atmosphere of all other (N-1) subsystems. $\mu_{1}^{e l}$ is the partial electrostatic potential of the ion ' $i$ ' in the given solution due to the contribution of the electrostatic interionic forces.strictly speaking, it would be wrong to identify this $\mu_{i}^{e l}$ with the electrical work done when we add a single ion " $i$ " to the particular subsystem in which " $i$ " is the central ion, as it is implicitly assumed by Guentelberg, (cf.ref[59]). This becomes evident when we consider Guentelberg's actual method of calculation of $\mu_{i} e l$. This consists of two steps: (a) Add the discharged ion " $i$ " to the particular subsystem; and (b) increase the charge of the central ion " $i$ " from zero to $e_{i}$. It is obvious that in this procedure we are not adding the ion " 1 " to the actually given system, (cf. eq. (5.10)). In order to calculate $\mu_{i}^{e l}$ correctly we have also to charge up the ion " $i$ " which are present in all other ( $\mathrm{N}-1)$ subsystems as the atmospher 1c ion. Consequently, the value of $\mu_{i}^{e l}$ calculated by the method of Guentelberg does not include the contribution of the ion " $\mathrm{I}$ " present in the remaining ( $\mathrm{N}-1$ ) subsystems.

In order to realize how difficult it would be to carry out Guentelberg's calculation correctly, let us write the correct expression for the infinitesimal 
Increase in the free energy of the given system due to an infinitesimalincrease of the charge of the ion " 1 ". It is given by

$$
\begin{aligned}
d F^{e l} & =\phi_{1}\left[v e_{1} ; x\left(v e_{i}, e_{j} s ; a_{i}\right)\right] e_{i} d v \\
& +\sum_{\substack{j=1 \\
\neq 1}}^{N-1} \phi_{j}\left[e_{j} ; x\left(v e_{i}, e_{j} s\right) ; r_{j i}\right] e_{i} d v
\end{aligned}
$$

Now $x$ depends on $e_{j}$ 's as well as on $v_{i}$. The second term shows that $\phi_{j}$ depends not on the constant $a_{i}$, but on the variable $r_{j i}$, the distance of the $1^{\text {th }}$ ion from the centre of the $j^{\text {th }}$ central ion. $\phi_{j}(r)$ here is the potential at the site of the Ion " $i$ " in the particular subsystem in which " $f$ " is the central ion. In order to get the full contribution of the ion " $i$ ", we have to integrate the expression (5.11) for $v$ varying from 0 to 1 and for the second term also $r_{j i}$ from $a_{j}$ to $\infty$. Obviously, it would be practically impossible to carry out this correctly, particularly if we consider the non-linear potential. Moreover, one should also note that in this method we are ignoring the volume of the particular ion " $i$ " and $X$ is affected unsymmetrically and the system violates the electroneutrality condition.

We can therefore conclude that $\mathrm{DH}$ method of simultaneous charging process is not only logically more satisfactory but it is also much easier to calculate the free energy.

(viii) Invalidity of Debye-Hueckel Theory As A Limiting Theory.

First, let us note that if one uses Boltzmann distribution in the field of electrostatic forces alone, one is implicitly using the concept of point charges. Consequently, as is well known, Gibbs' configuration integral diverges. Also the system becomes unstable and leads to physically absurd results, as had been proved before, (see refs. [8], [9], [55], [56]). One should particularly note that, as proved by GronwalI. [9], the situation is not changed by using the finite size of the ionic radius as the boundary condition for the PB equation. Physical 
Inconsistency of the original $\mathrm{DH}$ theory can also be proved from a very simple consideration, (cf Bagchi [7]). For example, in the case of 1-1 electrolyte we get for a central positive ion:

$$
\begin{aligned}
\mathrm{n}^{+}(\mathrm{r})+\mathrm{n}^{-}(\mathrm{r}) & =\mathrm{n}_{0}^{+} \exp -(\varepsilon \psi / k T)+\mathrm{n}^{-}{ }_{0} \exp +(\varepsilon \psi / k T) \\
= & \mathrm{n}_{0}^{+}+\mathrm{n}_{0}^{-}+\Delta>\mathrm{n}_{0}^{+}+\mathrm{n}_{0}^{-},
\end{aligned}
$$

since $\Delta$ is a positive quantity.

Thus we see that it leads to the absurd physical result, namely, the total number of lons becomes greater than that which is contained in the system. It might be noted that the modified DH theory does not suffer from such physical or mathematical inconsistencies.

Hence, it is neither mathematically nor physically justified to claim that the original DH theory is strictly valid as a limiting theory. At best, we can only accept the results derived from the limiting theory in the sense that the free energy is not appreclably dependent on the actual value of the fonic radius so far as the numerical magnitude is concerned, a conclusion which was drawn long ago by Halpern [55] from a different consideration. Nevertheless, the original $\mathrm{DH}$ theory in principle remains physically and mathematically inconsistent.

of course, this absurd result arises not from the inadequacy of Boltzmann distribution per se but because of the fact that we have neglected short range forces. If we take the short range forces into Boltzmann distribution we cannot use $\mathrm{PB}$ equation. We have to solve either a complicated nonlinear pde or face the intractable problem of evaluating the configuration integral. These technical difficulties can be overcome only by using the modified $\mathrm{DH}$ theory.

Finally, it should be noted that in spite of the invalidity of the original DH theory, the ion-atmosphere concept and the $\mathrm{DH}$ technique for calculating the free energy of the system remain valid not only for electrolytic solutions but 
also for fused salts. The exhaustive results obtained from modified DH theory, (see refs [21], [23]) for solutions of electrolytes and the results obtained from this new approach for fused alkali halides, (see Table I), conclusively demonstrate that the ion-atmosphere concept of Debye \& Hueckel and their technique for calculating the excess free energy remain as one of the outstanding achievements of equilibrium and non-equilibrium statistical mechanics. At the present state of our knowledge, it can be safely concluded that this technique offers us the only practically feasible method to calculate equilibrium and nonequilibrium properties not only of systems of charged particles but also of any classical system. Moreover, unlike all other methods this method remains physically and mathematically consistent for. all densities.

(ix) Ad Hoc Procedures To Improve Debye-Hueckel Theory.

From the very beginning it was generally recognized that for point charges $\mathrm{DH}$ theory would give divergent and mathematically meaningless results. Kramers [8] tried to avoid thisdivergent result by introducing arbitrarily a potential of the form $U\left(\vec{r}_{1}, \ldots \vec{r}_{N}\right)=D^{-1}{ }_{j} \sum_{k} e_{j} e_{k} g_{j k}$

$$
\text { where } \begin{aligned}
g_{j k} & =r_{j k}{ }^{-1}\left[1-\exp \left(-\lambda h_{j k}\right)\right] ; \\
h_{j k}{ }^{2} & =\frac{\left(x_{j}-x_{k}\right)^{2}+\left(y_{i}-y_{k}\right)^{2}+\left(z_{j}-z_{k}\right)^{2}}{\left(x_{j}+x_{k}\right)^{2}+\left(y_{j}+y_{k}\right)^{2}+\left(z_{j}+z_{k}\right)^{2}}
\end{aligned}
$$

and $\lambda$ was chosen to be a very large number. Berlin \& Montrol1[56] pointed out the drawbacks of Kramers' method and tried to eliminate them by introducing, again arbitrarily, the parameter $x^{2}$.

Mayer-McMillan cluster theory of ionic solutions is now considered (albeit wrongly) as a rigorous theory. A closer examination of the foundation of this theory (cf. Friedman [61]) would reveal that this theory is also an ad hoc theory tailored to avoid the difficulties of the evaluation of the 
configuration integral Involving only Coulomb forces. Mayer replaced the Coulomb potential by $e^{-\alpha r} / r$, the Yukawa (1.e. Iinear Debye) potential, and taking $\alpha>0$. It is clear that Ursell-Mayer function for the pairwise interaction of Coulomb potential, $\left(\begin{array}{llll}\sum_{i j} & u=\sum_{i j} & e_{i} e_{j} \\ r_{i j}\end{array}\right)$, cannot lead to a potential function of this type. The potential of the form $\bar{e}^{\alpha r} / r$ can be obtained either from the type of meson equation or PB equation of DH theory. Consequently, within the scope of Mayer's cluster theory there is no theoretical justification for introducing such a potential. Further, DH limiting law is obtained by letting $\alpha \rightarrow 0$ which however makes the theory mathematically inconsistent.

Consequently, one can definitely assert that all these ad hoc theoretical recipes to derive thermodynamic properties of fonic solutions have no secure theoretical foundation within the formalisms of these theoretical investigations. Therefore, all these theories cannot be accepted as rigorous approaches to the problem in question. There is no doubt that the actual average potential of such systems is given by a term like $\bar{e}^{\alpha r} / r$ in the linear approximation (see Bagchi $\&$ Plischke [24]) and the parameter $\alpha$ plays a significant role in such systems, but they cannot be derived theoretically from these theories. Consequently, in theoretical treatments of Kramers, Berlin \& Montroll, Mayer and others, such terms had to be introduced as convenient recipes. Rigorous theoretical formulation of the problem can be achieved by evaluating the configuration integral which incorporates directly, besides Coulomb forces, all other forces, in particular, short range repulsive forces. But as we have noted before, the problem would then be, in all probability, an intractable one. Therefore, the only feasible method, which is physically as well as mathematically consistent, is to utilize this new approach in which the exclusion volumes, determined by the repulsive forces, are directly incorporated in the distribution function itself. 
Apart from this fundamental question of mathematical inconsistency, it has been known from the beginning that the original DH theory cannot reproduce even qualitatively the observed properties of concentrated solutions. Consequently, ignoring the fundamental difficulties of $\mathrm{DH}$ theory, many workers tried to obtaln better agreement with experimental values by supplementing original $\mathrm{DH}$ theory with various arbitrary physical assumptions and models. One of the most persistent as well as old model is to use the concept of ion-assoclation. But it is known that even in the solid state ions exist as separate entities. Further, there is no theoretical justification to consider the consequences of Ionassociation separately and outside the ion-atmosphere theory. It is interesting to note that Gronwall [9] also came to the same conclusion. From the standpoint of ion-atmosphere theory, ion-association means that opposite ions are tightly bound together due to the large ion-atmospheric potential on the surface of the ions. The mean activity coefficient becomes infinitely small. For example, actual exact calculation shows that for 3-3 electrolytes for $a=0.5 \mathrm{~A}^{\circ}$ at $0.01 \mathrm{~N}$ the mean activity coefficient becomes as low as $0.56 \times 10^{-17}$, (see Bagchi [23]). Some workers attempted to get closer agreement with experimental values of activity coefficients by calculating the additional contributions due to the hydration energy of the ions. But if we note that ions are already hydrated in infinitely dilute solutions and it is only the heat of dilution and not the hydration energy which can contribute to the free energy of concentrated ionic solutions, a quantity whose magnitude cannot explain the difference between the activity coefficients and those calculated from the original DH theory, it will be clear that one cannot accept any such ad hoc treatment as a satisfactory basis for developing a logical and mathematically consistent theory of solutions of electrolytes.

Onsager [50] and van Rysselberghe [62] tried to incorporate an additional term due to short range forces, the socalled "co-volume effect", in the expression 
for the free energy of the system. Basically, it does not eliminate the inconsistencles inherent in the original $\mathrm{DH}$ theory. For a rigorous treatment, one should incorporate this short range force in the configuration integral and then calculate the excess free energy due to the coulomb potential and short range force.

\section{(x) Modified Debye-Hueckel Theory}

As noted before, the only feasible method of calculating the electrostatic free energy, which is mathematically and physically consistent, is to use the concept of Ion-atmosphere and DH technique with a new distribution function which incorporates the exclusion volume directly in the formula (cf.eq. 4.27). The consistency is due to the fact that by this method one has taken into account the replusive forces in a most convenient way so that one can also use the modIfied $P B$ equation.

Of course, it is still an approximation, particularly for the case of lonic solution, mainly due to the presence of moter molecules, because we have neglected polarization forces. But to a large extent this drawback is compensated by taking the macroscopic dielectric constant into consideration. For fused salts, this is adequate enough. For ionic solutions, one has to choose correctly the effective fonic radius and the exclusion volumes. Once they are properly chosen both equilibrium and non equilibrium properties can be calculated satisfactorily without incorporating any ad hoc recipes. Consequently, in principle the modified DH theoy can be considered as the only fundamental consistent theory for systems of charged particles which is at the same time practically feasible and can yield reliable results in spite of legitimate approximations involved in the theory. Also, in principle these limitations can be overcome if we use a pertinent nonlinear pde relevant for all types of forces. For details regarding the ion-atmosphere theory of electrolytic solutions, modified as well as the original DH theory, see the next issue of this journal. 


\section{CONCLUSIONS}

All known methods of statistical mechanical investigations, for both equilibrium and non-equilibrium phenomena, lead to mathematical divergences at high densities even for simple systems. For a system of charged particles interacting with Coulomb forces one cannot even formulate the theory in a mathematically consistent way, if one follows conventional methods. These difficulties and inconsistencies are not due to the inadequacies of the basic principles of statistical mechanics. They also do not arise from the fact that certain important structure parameters, for example., the presence of physical micro-clusters in a dense system, had not been incorporated in the framework of the theory. They are mainly due to computational techniques. For example, in the integration processes one does not take into account explicity the fact that every particle has a "hard core" within which no other particle can ever enter. At the present state of our knowledge, it is difficult to see how one can take into account' all possible configurations of these moving infinite potential barriers in the evaluation of Gibbs' configuration integral. As a result, although it is obvious that an equation of state exists for any substance at any density, the conventional methods do not permit us to calculate the equation of state even for a simple system like hard spheres at high densities. Consequently, the virial coefficients calculated from these methods cannot be accepted as correct.

Based on the secure foundations of statistical mechanics and by taking into account explicity the presence of these hard cores, an alternative approach has been proposed here which not only overcomes all these difficulties but also yields reliable physical results, for both thermodynamic and transport properties of any classical system,including a system of charged particles. The striking success of this approach is mainly due to the fact that in this method the phase-space ( $\mu$-space) is separated into configuration space and momentum space and the physical space is partitioned into elementary cells, which are exclusion volumes of Boltzmann. 
The average potential of the system is then calculated from a relevant partial differential equation. The distribution of particles around a central one incorporates directly these exclusion volumes and this average potential. In order to derive this new distribution function, the particles are distributed independently into these elementary cells of the physical space with the obvious restriction that a cell either remains vacant or is occupied by one particle only. Consequently, the distribution function has a formal resemblance with Fermi distribution of quantum statistics. But it should be noted that contrary to quantum statistics here the distribution function can be expressed in terms of the potential energy alone. Also, as expected, this new distribution function leads to classical Boltzmann distribution in the potential field when the cell sizes can be neglected.

This new approach thus circumvents the difficulties encountered in the original Boltzmann's method of counting complexions in $\mu$-space as well as in the evaluation of Gibbs' configuration integral. For equilibrium systems it yields directly the partition function due to kinetic energies of the individual particles which contains explicity the hard cores of the particles. The "excess" free energy due to the potential field can then be obtained separately from the average potential of the systems in several ways. Thus the thermodynamic problem is solved completely.

For calculating transport coefficients, the problem also becomes much simpler. The particle always moves underthe influence of aninternal field and its motion is hindered only by"direct collisions"with other hard spheres. Consequently, it is sufficient to take into account only binary collisions, since it is very unlikely that moving particles, considered as hard spheres, would suffer simultaneous multiple collisions.

It appears that at the present stage of our knowledge, this new approach offers us the only feasible way to tackle the problems of statistical mechanics (both 
equilibrium and nonequilibrium) of any classical system at any density in a mathematically consistent and rigorous manner.

\section{REFERENCES}

[1] BAGCHI, S.N. A new approach to statistical mechanics of classical systems. Part I. Hard Spheres. Physica (1980). Communicated.

[2] BAGCHI, S.N. Do. Part II. Mixture of Hard Spheres, Physica (1980). Communicated.

[3] BAGCHI, S.N. Statistical mechanics of fused ionic salts. Phys. Letters, 74A, (1979), 271-274.

[4] BOgOliUbov, N.N. Problems of a dynamical theory in statistical physics, in Studies in Statistical Mechanics, vol 1, (1962), 1-116. North Holland Pub. Co., Amsterdam.

[5] COHEN, E.G.D. On the non-existence of density expansions for the transport coefficients in classical gases in statistical mechanics: Foundations and Applications, Proc. IUPAP meeting, Copenhagen. Ed. Thor. A. Bak, W.A. Benjamin, New York. (1967), 291-312.

[6] CHAPMAN, S. \& COWLING, T.G. The Mathematical Theory of Non-Uniform Gases. Third Edition. Camb. U.P. (1970).

[7] BAGCHI, S.N. Statistical validity of the ion-atmosphere concept and its application in calculating the pair-distribution function in many body systems. Proc. Int. Symp. on Statistical Physics, Ed. M. Dutta, Calcutta University, (1974), 49-52.

[8] KRAMERS, H.A. Investigations on the free energy of a mixture of ions. Proc. Royal Acad. Amsterdam, 30, (1927), 145-158.

[9] GRONWALL, T.H., LA MER, V.K. \& SANDVED, K. Veber des Einfluss der sogenanten hoeheren Glieder in der Debye-Hueckelschen Theorie der Loesungen starker Elektrolyte. Phys. Zeit, 29. (1928), 358-393.

[10] DEBYE, P \& HUECKEL, E. Zur Theorie der Elektrolyte. Phys. Zeit, 24, (1923), 185-206.

[11] FRENKEL, J. Kinetic Theory of Liquids. Oxford U.P. (1946).

[12] EYRING, H.\& JHON, M.S. Significant Liquid Structures, John Wiley \& Sons, New York, (1969).

[13] MUENSTER, A. Statistical Thermodynamics, Vo1. 2, Ch 16, Springer Verlag, New York, (1974).

[14] DUTTA, M. On a treatment of imperfect gas after Fermi's model. Proc. Nat1. Inst. Sciences, India, 13, $(1947), 247-52 ; 14,(1948), 163-168 ; \underline{17}, \overline{(1951)}$, 27-37; 445-466. 
[15] BAGCHI, S.N. The structure of liquids as revealed by the analysis of their radial distribution functions. Acta. Cryst. A28, (1972), 560-571.

[16] HOSEMANN, R. \& BAGCHI, S.N. Direct Analysis of Diffraction by Matter, North Ho1land Pub.' Co., Amsterdam, (1962).

[17] BAGCHI, S.N.' Kinematic theory of diffraction by matter of any kind and the theory of liquids. Adv. Phys, 19, (1970), 119-173.

[18] BAGCHI, S.N. A new equation for strong electrolytes, J. Ind. Chem. Soc. 27, (1950), 199-203; 204-213.

[19] DUTTA, M. \& BAGCHI, S.N. On the distribution of ions in solutions of strong electrolytes, Ind. J. Phys. 24, (1950), 61-66.

[20] BAGCHI, S.N. On strong electrolytes in solutions, Naturwiss. 39, (1952), 299-300; Veber die Verteilung von Ionen in Loesungen starker Elektrolyte, Z. Elektrochem. 57, (1953), 138-140.

[21] DUTTA, M. \& SENGUPTA, M. A theory of strong electrolytes in solution based on a new statistics: Activity coefficients. Proc. Nat1. Inst. Sciences, India, 20, (1954), 1-11

SENGUPTA, M. A theory of strong electrolytes in solution: Activity coefficients. J. Ind. Chem. Soc. 45, (1968), 1133-1150.

EIGEN, M. \& WICKE, E. The thermodynamics of electrolytes at higher concentrations. J. Phys. Chem. 58, (1954), 702-714.

FALKENHAGEN, H. Elektrolyte, Zweite Auflage, S. Hirzel Verlag, Leipzig, (1953).

FALKENHAGEN, H. Bemerkungen zu einigen neueren Ergebnissen der Theorie starker Elektrolyte. Proc. Int. Symp. on Transport Processes in Statistical Mechanics, Brussels. Ed. I. Prigogine. Interscience, New York, (1958).

[22] WICKE, E. \& EIGEN, M. Zur Theorie der starken Elektrolyte, Naturwiss, 38, (1951), 453-459.

[23] BAGCHI, S.N. Ion-atmosphere theory of strong electrolytes. Part I. DebyeHueckel theory, Parts II and III. Modified Debye-Hueckel theory, (1974), (unpublished).

[24] BAGCHI, S.N. \& PLISCHKE, M. An analytic solution of the nonlinear differential equation, $\Delta \lambda=f(\lambda)$, J. Ind. Chem. Soc. 45, (1968), 923-935.

[25] BAGCHI, S.N. The role of diffraction theory in the phenomenon of phase transitions. Lecture delivered at the Second Int. Conf. on Small-angle scattering, Graz, (Austria), (1970), 1-96, (unpublished). Unified kinematic theory of diffraction and its role in biological structures, in the theoy of liquids and in phase transitions. Lectures delivered at the University' of Buffa1o, New York, (1971), 1-58, (unpublished).

[26] BAGCHI, S.N. \& SUPPLE, J.P. Structural parameters of inert liquids. Part I: Liquid Helium, Neon and Xenon. Phys. Chem. Liq. 8, (1979), 223-234.

[27] BAGCHI, S.N. \& SUPPLE, J.P. Do. Part II. Liquid Argon (1980). Communicated. 
[28] BAGCHI, S.N. Some remarks about first order phase transformation and the critical state. Proc. Int. Symp. on Statistical Physics, Ed. M. Dutta. Calcutta University, (1974), 197-200.

[29] HOSEMANN, R. \& BAGCHI, S.N. Begruendung einer Algebra physikalisch beobachtbarer Funktionen mittels Faltungs operationen. Part I. Praezisionen, Funktionkomplex, Punkt functionen, Fourier-transformation eines komplexes. Integro-differential operator. Z. Phys. 135, (1953), 50-84. Part II Fouriertransformation von Potenzrethen und Multipol-reihen, Zusammenhang mit Laplaceund Mellin-transformation, Eine Erweiterung der Funktion Theorie, $\underline{Z}$. Phys. 137, (1954), 1-30.

[30] GHOSH, P.K. On the mathematical foundations of physically observable functions. Bul1. Cal. Math. Soc. 49, (1957), 25-28. see also: ref. [16], 674-687.

[31] TER farr, D. Elements of Statistical Mechanics, Holt, Rinehart and Winston, New York, (1961).

[32] HILL, T.L. Statistical Mechanics, McGraw Hi11, New York (1956).

[33] VAN HOVE, L. Quelques propriétés générerales de l'integrale de configuration d'un systeme de particules avec interaction. Physica, 15, (1949), 951-961.

[34] YANG, C.N. \& LEE, T.D. Statistical theory of equation of state and phase transitions I. Theory of Condensations. Phys. Rev. 87, (1952), 404-409.

[35] HILL, T.L. Thermodynamics of Small Systems. Parts I \& II. W.A. Benjamin, New York, (1963 \& 1964).

[36] CHERNOFF,P.R. \& MARSDEN, J.E. Properties of Infinite Dimensional Hamiltonian Systems. Lecture Notes in mathematics, vol. 425. Springer Verlag, New York, (1974).

[37] SINAI, Ya.G. Ergodic theory in Boltzmann Equation, Springer Verlag, New York. (1973), 575-608.

[38] FOWLER, R.H. Statistical Mechanics, Camb. U.P. (1966).

[39] JEANS, J.H. The Dynamic Theory of Gases. Fourth edition. Dover Publications, New York, (1954).

[40] LINDEMANN, F.A. Ueber die Berechnung molekularer Eigen-frequenzen. Phys. Zeit. 11, (1910), 608-612.

[41] MIKOLAJ, P.G. \& PINGS, C.J. in X-ray scattering studies of simple fluids, Simple Dense Fluids, Ed. H.L. Frisch and Z.N. Salsburg, Academic Press, New York, (1968), 42-61.

[42] THOMAS, J.E. \& SCHMIDT, P.W. X-ray studies of critical opalescence in argon. J. Chem. Phys. 39, (1963), 2506-2516.

[43] SCHROEDINGER, E. The statistica1 law in nature. Nature, $153,(1944)$, 704705 . 
[44] SINAI, Ya. G. Introduction to Ergodic Theory, Princeton U.P., Princeton, N.J. (1976).

[45] ARNOLD, V.I. \& AVEZ, A. Ergodic Problems of Classical Mechanics, W.A. Benjamin, New York (1968).

[46] MAZO, R.M. Statistical Mechanical Theories of Transport Processes, Pergamon Press, Oxford, (1967).

[47] TOLMAN, R.C. The Principles of Statistical Mechanics. Oxford U.P. (1955).

[48] SCHROEDINGER, E. Statistische Thermodynamik. Johan Ambrosius Barth, Leipzig, (1952).

[49] WILSON, A.H. Thermodynamics and Statistical Mechanics. Camb. O.P., (1960).

[50] ONSAGER, L. Theorles of concentrated electrolytes. Chem. Rev. 13, (1933), 73-89.

[51] BRILIOUIN, L. A comparison of the different statistical methods applied to quantum problems in Selected Papers on Wave Mechanics by L. de. Broglie and i. Brillouin. Blackie and Sons, London, (1928), 139-150.

[52] SABA, M.N. \& BOSE, S.N. On the influence of finite volume of molecules on the equation of state. Phil. Mag. 36, (1918), 199-202.

[53] SAFA, M.N. \& SRIVASTAVA, B.N. A Treatise on Heat. Fourth Edition. The Indian Press, Allahabad, (1958), 423.

[54] FELLER, W. An Introduction to Probability Theory and Its Applications. Vol. I, John Wiley and Sons, New York, Second Edition, (1966), 41.

[55] HALPERN, 0 . On the statistical basis of the theory of electrolytes. J. Chem. Phys. 2, (1934), 85-93.

[56] BERLIN, T.H. \& MONTROLI, E.W. On the free energy of a mixture of lons: An extension of Kramer's theory. J. Chem. Phys. 20, (1952), 75-84.

[57] FOWLER, R.H. Strong electrolytes in relation to statistical theory, in particular the phase integral of Gibbs. Trans. Farad. Soc. 23, (1927), 434443.

[58] KIRKWOOD, J.G. On the theory of strong electrolyte solutions. J. Chem. Phys. 2, (1934), 767-781.

[59] FOWLER, R.H. \& GUGGENHEIM, E. Statistical Thermodynamics, Camb. U.P. (1956).

[60] SENGUPTA, M. On a consistency test of the theories of strong electrolytes. Ind. J. Phys. 27, (1953), 628-632.

[61] FRIEDMAN, H. L. Ionic Solution Theory, Interscience, New York, 1962.

[62] VAN RYSSELBERGHE, P. \& EISENBERG, S. Activity coefficients in concentrated aqueous solutions of strong electrolytes described by a formula containing the mean ionic diameter as single parameter. J. Phys. Chem. 61, (1939), 3030-3037. 


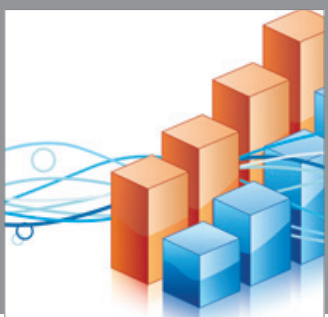

Advances in

Operations Research

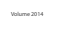

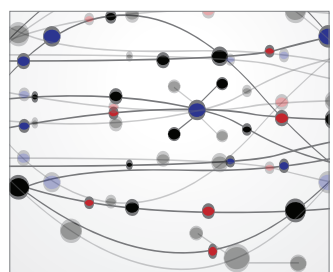

\section{The Scientific} World Journal
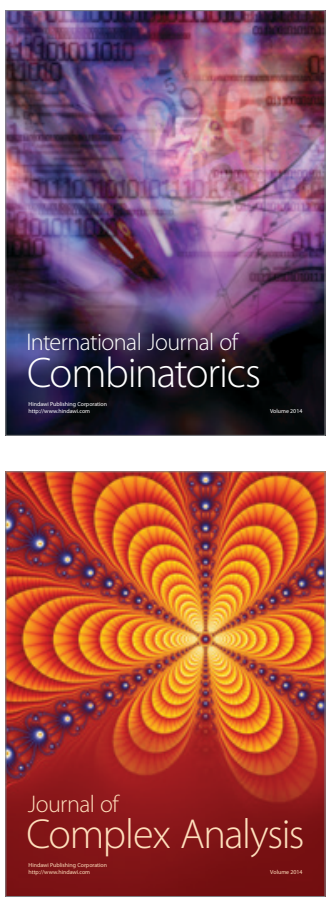

International Journal of

Mathematics and

Mathematical

Sciences
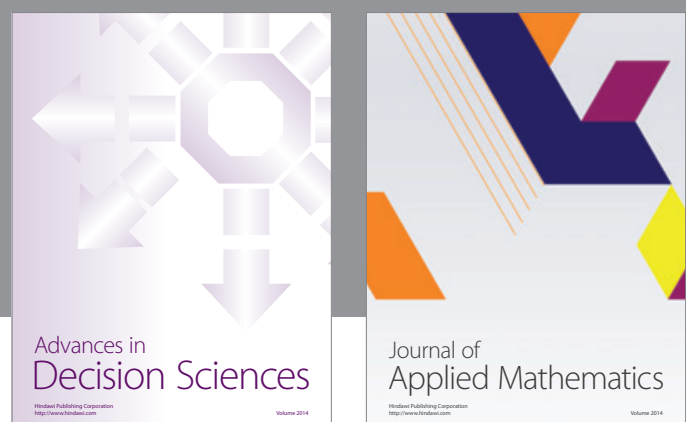

Journal of

Applied Mathematics
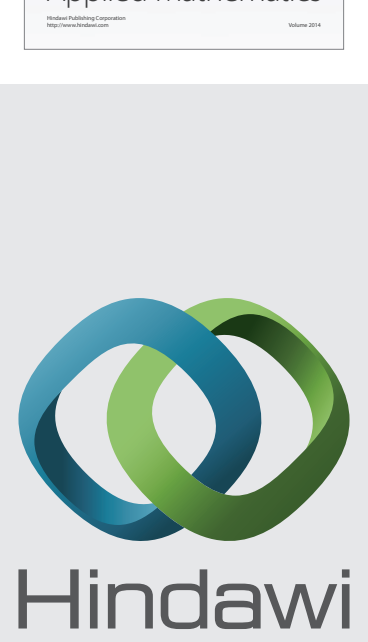

Submit your manuscripts at http://www.hindawi.com
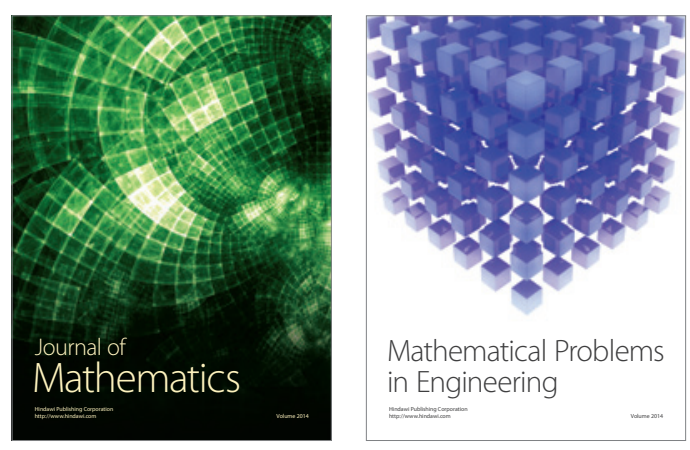

Mathematical Problems in Engineering
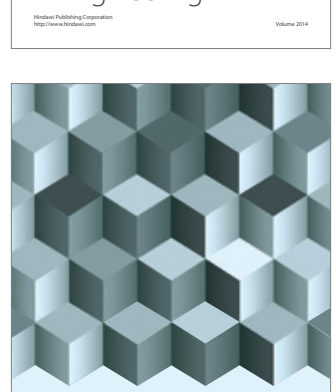

Journal of

Function Spaces
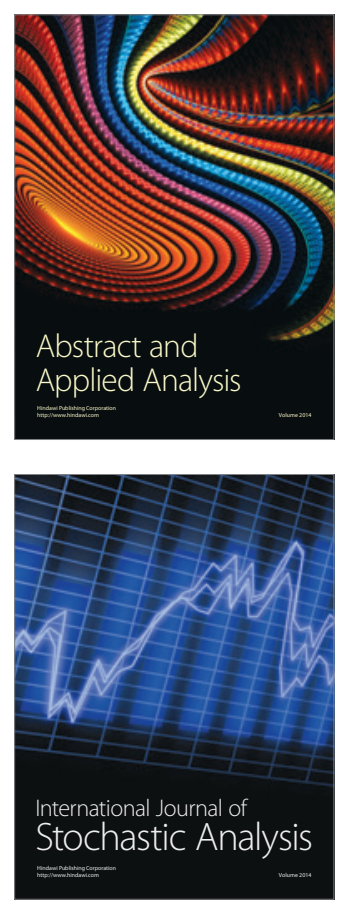

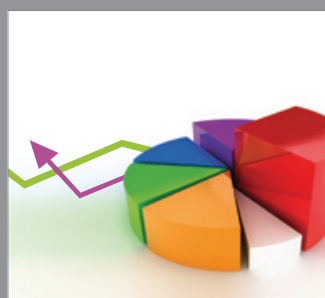

ournal of

Probability and Statistics

Promensencen
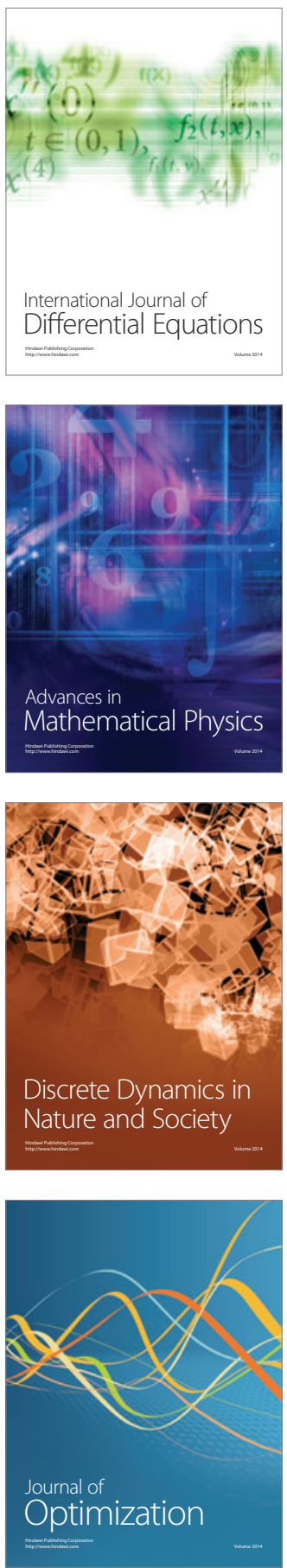